\title{
Optimized ratiometric calcium sensors for functional in vivo imaging of neurons and $\mathrm{T}$ lymphocytes
}

\author{
Thomas Thestrup $^{1,2}$, Julia Litzlbauer ${ }^{1}$, Ingo Bartholomäus ${ }^{1}$, Marsilius Mues ${ }^{1}$, Luigi Russo ${ }^{3}$, Hod Dana ${ }^{2}$, \\ Yuri Kovalchuk ${ }^{4}$, Yajie Liang ${ }^{4}$, Georgios Kalamakis ${ }^{4}$, Yvonne Laukat ${ }^{3}$, Stefan Becker ${ }^{3}$, Gregor Witte ${ }^{5}$, \\ Anselm Geiger ${ }^{1}$, Taylor Allen ${ }^{6}$, Lawrence C Rome ${ }^{7,8}$, Tsai-Wen Chen ${ }^{2}$, Douglas S Kim ${ }^{2}$, Olga Garaschuk ${ }^{4}$, \\ Christian Griesinger ${ }^{3}$ \& Oliver Griesbeck ${ }^{1,2}$
}

The quality of genetically encoded calcium indicators (GECIs) has improved dramatically in recent years, but high-performing ratiometric indicators are still rare. Here we describe a series of fluorescence resonance energy transfer (FRET)-based calcium biosensors with a reduced number of calcium binding sites per sensor. These 'Twitch' sensors are based on the C-terminal domain of Opsanus troponin C. Their FRET responses were optimized by a large-scale functional screen in bacterial colonies, refined by a secondary screen in rat hippocampal neuron cultures. We tested the in vivo performance of the most sensitive variants in the brain and lymph nodes of mice. The sensitivity of the Twitch sensors matched that of synthetic calcium dyes and allowed visualization of tonic action potential firing in neurons and high resolution functional tracking of T lymphocytes. Given their ratiometric readout, their brightness, large dynamic range and linear response properties, Twitch sensors represent versatile tools for neuroscience and immunology.

Imaging with GECIs has become a widely used method in physiology and neuroscience ${ }^{1-3}$. According to readout mode, the design of the sensors has followed two different pathways, leading to single-wavelength sensors and FRET-based ratiometric sensors ${ }^{4-8}$. Among the most popular single-wavelength sensors are the G-CaMPs ${ }^{9-13}$, R-CaMPs ${ }^{14}$ and GECOs ${ }^{15}$. FRET sensors include yellow cameleon 3.60 (refs. 16,17), D3cpv ${ }^{18}$, yellow cameleon Nano ${ }^{19}$ and TN-XXL ${ }^{20}$.

Quantification by ratiometric FRET imaging is more accurate than single-channel measurements and may be more suitable for long-term functional imaging studies, as it is less influenced by changes in optical path length, excitation light intensity and indicator expression level and by tissue movement and growth during development. In addition, FRET indicators are substantially brighter than single-wavelength sensors at rest, allowing better identification of expressing cells and their subcellular structures.
Another practical feature of FRET-based indicators is their ability to measure basal $\mathrm{Ca}^{2+}$ levels within cells, for example, to distinguish between resting and continuously spiking neuronssomething that cannot easily be achieved with single-wavelength indicators ${ }^{21}$. Increased basal $\mathrm{Ca}^{2+}$ levels within the brain are also observed at the onset of neurodegenerative processes, and ratiometric FRET calcium imaging has been used in these conditions to monitor disease progression ${ }^{22,23}$. Moreover, ratiometric indicators are advantageous for monitoring calcium in motile cells.

Both calmodulin and troponin $\mathrm{C}(\mathrm{TnC})$, the calcium binding proteins within the various GECIs, consist of two globular domains connected by a central linker ${ }^{24,25}$. Each domain possesses two calcium-binding EF hand motifs. Thus, currently available GECIs are highly nonlinear sensors binding up to four calcium ions per sensor. Identification of a smaller calciumbinding domain with fewer binding sites could help to reduce buffering during long-term chronic GECI expression ${ }^{26}$, make the sensor smaller and further minimize the risk of cytotoxicity. It may also help to simplify response properties and facilitate the biophysical modeling of sensor behavior.

Here we report several improvements of FRET-based calcium sensors for in vivo imaging. First, we identified a minimal calcium binding motif based on the C-terminal domain of $\mathrm{TnC}$ with only two or one remaining calcium binding sites per sensor molecule, thus reducing the overall calcium buffering of the sensors. Second, by sampling TnCs from various species we identified a new TnC variant from the toadfish Opsanus tau, which offered the possibility of generating minimal domains with high-affinity calcium binding. Third, we used a large-scale, two-step functional screen to optimize the FRET changes in the sensor by linker diversification. This approach allowed us to identify Twitch sensors with a superior FRET change and may become useful for optimizing other types of FRET sensors. Finally, we improved brightness and photostability of the sensors by incorporating enhanced donor and acceptor fluorescent protein variants. Taken together, these

\footnotetext{
${ }^{1}$ Max Planck Institute of Neurobiology, Martinsried, Germany. ${ }^{2}$ Janelia Farm Research Campus, Howard Hughes Medical Institute, Ashburn, Virginia, USA. ${ }^{3}$ Max Planck Institute for Biophysical Chemistry, NMR-based Structural Biology, Göttingen, Germany. ${ }^{4}$ Institute of Physiology II, University of Tübingen, Tübingen, Germany. ${ }^{5}$ Gene Center, Department of Biochemistry, University of Munich, Munich, Germany. ${ }^{6}$ Oberlin College, Oberlin, Ohio, USA. ${ }^{7}$ Department of Biology, University of Pennsylvania, Philadelphia, Pennsylvania, USA. ${ }^{8}$ The Whitman Center, Marine Biological Lab, Woods Hole, Massachusetts, USA. Correspondence should be addressed to O. Griesbeck (griesbeck@neuro.mpg.de).
}

RECEIVED 15 JUNE 2013; ACCEPTED 29 NOVEMBER 2013; PUBLISHED ONLINE 5 JANUARY 2014; D0I:10.1038/NMETH.2773 
a
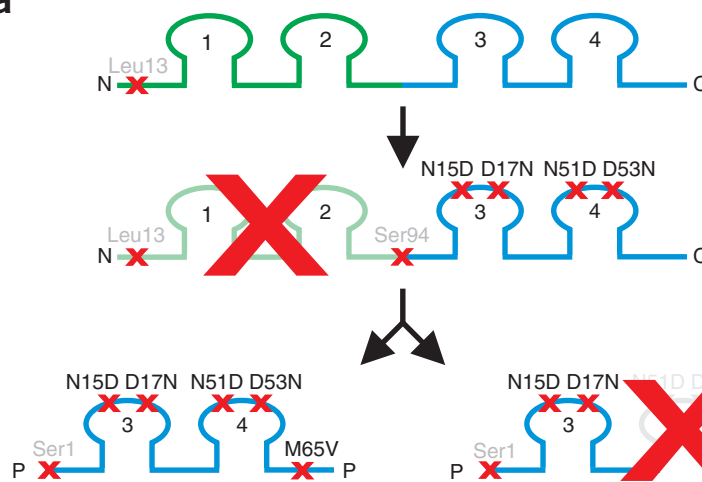

Figure 1 | Minimizing the calciumbinding domain in Twitch sensors. (a) Schematic representation of $\mathrm{TnC}$ as a serial arrangement of four calcium-binding EF hands, depicted as
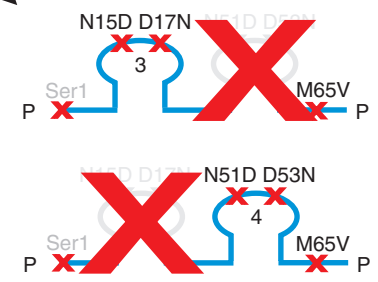

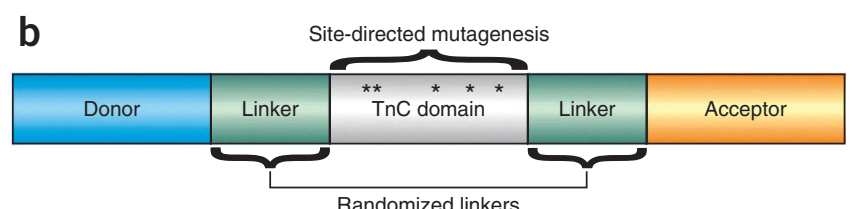

C

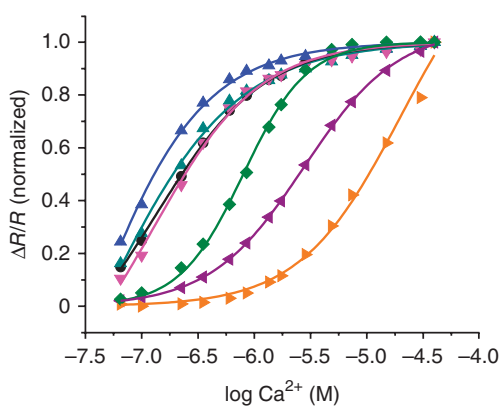

TN-XXL

Twitch-1

- Twitch-2

- Twitch-2B

$\checkmark$ Twitch-3

Twitch-4

Twitch-5

loops (1-4). Removal of the N-terminal domain (green) creates a minimal calcium-binding domain (blue). Mutations (indicated by small ' $x$ ') are introduced into the EF hands to render them specific for calcium over magnesium. Within the minimal domain, one of the remaining EF hands can be crippled by mutagenesis to generate 'single-hand' sensors in which response properties are dominated by calcium binding to the remaining binding site. (b) Schematic representation of Twitch calcium sensors. The minimal domain is sandwiched between a donor and an acceptor fluorescent protein connected by amino acid linkers. Linker sequences can be randomized and screened for optimal calcium-induced FRET change. In addition, other sites within the minimal domain are targeted by site-directed mutagenesis to optimize sensor properties. (c) Calcium-affinity titrations of selected Twitch calcium biosensors (Supplementary Table 3).

improvements resulted in FRET-based calcium sensors that have sensitivities similar to synthetic calcium indicators and modern single-wavelength GECIs but that allow the valuable possibility of ratiometric imaging.

\section{RESULTS}

\section{Design of minimal domains for calcium sensing}

Calmodulin and TnC both consist of two globular domains that have different calcium binding properties and distinct biological function $24,25,27$. The dual-domain organization suggested to us that it might be feasible to generate sensors consisting of single domains instead of the full calcium-binding protein. The overall strategy that we followed to reduce GECI calcium binding sites is depicted in Figure 1. We found that the $\mathrm{N}$-terminal regulatory domain of TnC resulted in sensors that showed only small FRET changes in response to calcium. Therefore we focused on the C-terminal domains, which in our hands were responsible for almost all the conformational changes seen in the native protein in response to calcium. Domains isolated from the chicken skeletal muscle troponin $\mathrm{C}^{20}$ did not show sufficiently high affinity for calcium. We thus sampled the sequence diversity of TnC genes from various species and found that $\mathrm{TnC}$ derived from swim bladder and white muscle of $O$. tau (tsTnC) (Supplementary Figs. 1 and 2) allowed constructing minimal domain sensors with high-affinity calcium binding $\left(K_{\mathrm{d}}=140-400 \mathrm{nM}\right)$. We tested a series of truncations to the C-terminal domain, searching for variants that would optimally fit the calcium binding domain into the FRET sensor. The fragment we chose ranged from Ser93 to Gln161. We subsequently changed the residue numbering of wild-type tsTnC in our constructs for simplicity, renumbering Ser93 as Ser1. Additional point mutations into the original EF hands 3 (N15D, D17N) and 4 (N51D, D53N) were introduced to minimize magnesium binding ${ }^{28}$ (Fig. 1a,b). To generate lower-affinity sensors such as Twitch-4 and Twitch-5, we created a small library of sensors with amino acid insertions into EF hand 3 or 4 (Figs. 1a and 2). These mutants possessed only one functional EF hand with calcium affinities of $K_{\mathrm{d}} 2.8 \mu \mathrm{M}$ to $250 \mu \mathrm{M}$ (Fig. 1c). FRET signal change was initially improved by screening a small library of sensors with polyproline linkers inserted at the $\mathrm{N}$ and $\mathrm{C}$ termini of the domain (Supplementary Fig. 3). A minimal domain with a flanking proline on each side and a serendipitous beneficial mutation M65V that increased fluorescence ratio change led to the first prototypical minimal domain sensor, which we called Twitch-1. Twitch-1 had a maximal ratio change of $\sim 400 \%$ and a $K_{\mathrm{d}}$ of $250 \mathrm{nM}$ (Fig. 1c).

\section{Structural basis of Twitch calcium sensors}

For the development of Twitch sensors, we investigated the properties of different prototypes by solution NMR spectroscopy and small-angle X-ray scattering (SAXS). The solution NMR structural characterization was performed with the minimal C-terminal lobe domain of tsTnC in the calcium-loaded and calcium-free forms (the latter in presence of magnesium) (Supplementary Fig. 4). When calcium was present, ts TnC showed well-dispersed resonances in its ${ }^{1} \mathrm{H}_{-}{ }^{15} \mathrm{~N}$ HSQC spectra (Supplementary Fig. 4). A nearly complete assignment of backbone and $\mathrm{C} \beta$ chemical shifts was obtained by using standard triple-resonance experiments. Secondary structure elements were initially identified by the analysis of the chemical shift index ${ }^{29}$, and then confirmed by ${ }^{3} \mathrm{JH}_{\mathrm{N}} \mathrm{H} \alpha$ coupling values (Supplementary Fig. 4). The structure calculation for the protein was performed using a considerable number of structural restraints (Supplementary Table 1). A high-quality structure was obtained (Fig. 2a-c) consisting of a well-defined globular domain (r.m.s. deviation ${ }_{\mathrm{bb}}{ }^{2-65}=0.90 \AA$ ) with the typical topology known from the C-terminal domain of chicken skeletal $\operatorname{TnC}^{30}$ (Supplementary Fig. 5) with four helices ( $\alpha 1$, Glu2-Phe12; $\alpha 2$, Arg22-Thr32; $\alpha 3$, Asp38-Ser48; 
a

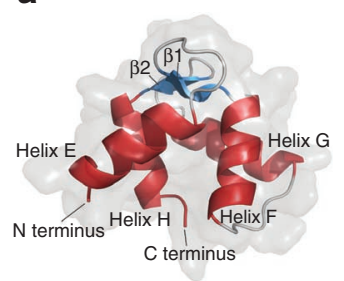

b

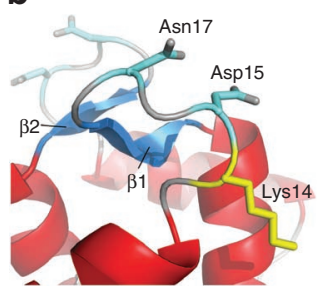

C

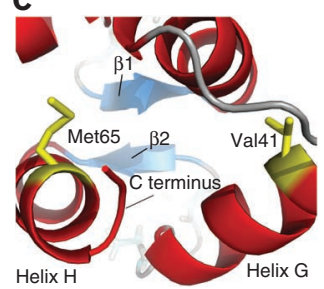

Figure 2 | Structural characterization of Twitch calcium sensors. (a) High-resolution NMR structure of the minimal calcium-binding domain based on the C-terminal lobe of TnC from 0. tau. (b) Close-up view of the calcium binding EF hand 3 and short $\beta$-sheet stretches. Sites of mutated residues within the calcium chelating loop to block magnesium binding (N17D, D15N) are indicated, as well as the position of another residue flanking EF hand 3 mutated to enhance FRET change (K14F) in some Twitch variants. (c) Close-up view of helices $\mathrm{H}$ and $\mathrm{G}$ with the indication of two other sites mutated to enhance FRET change (M65V, V41P). (d) Shape reconstruction of two Twitch sensors by SAXS. Shown are Twitch-1 and, for comparison, Twitch-0, an early construct with high basal FRET and small calcium-induced ratio change, at zero calcium (calcium free) and calcium saturation (calcium loaded). Multi-domain modeling in CORAL using high resolution structures of the calcium-loaded minimal domain as well as CFP (PDB 2WSN) and Citrine (PDB 3DPX) were used to localize the proteins within the SAXS structures. Shown are views from three angles. Twitch-1 changes from an extended structure, in which the fluorescent protein increased FRET. (e) Corresponding emission spectra of Twitch- 0 and Twitch-1 at zero calcium and calcium saturation.

$\alpha 4$, Phe58-Val68) and the two calcium binding EF hands 3 and 4 connected by a short antiparallel $\beta$-sheet $(\beta 1$, Phe19-Ile20Asp21; $\beta 2$, Arg55-Ile56-Val57). The $\mathrm{N}$ and the $\mathrm{C}$ terminus are close together, at a distance of only $15 \AA$. By contrast, the magnesium-loaded form in the absence of calcium is fully unfolded, which places the $\mathrm{N}$ and $\mathrm{C}$ termini at a distance of $5.2 \mathrm{~nm}$, on average, assuming a random coil distribution of conformations similar to ubiquitin ${ }^{31}$.

SAXS allowed us to monitor the shape and conformational change of the biosensor Twitch-1 including the attached fluorescent proteins CFP and cpCitrine (Fig. 2d,e). We obtained results on molecular geometry parameters such as radius of gyration $\left(R_{\mathrm{g}}\right)$, and maximum particle diameter $\left(D_{\max }\right)$, of the proteins, with approximations of folding status and conformation (Supplementary Table 2 and Supplementary Fig. 6). Algorithms for multidomain modeling ${ }^{32}$ using the high-resolution structures of the calcium-loaded minimal domain (Fig. 2a) and available structures for the fluorescent proteins permitted us to generate speculative fits of the probable localization of the proteins within the SAXS structure (Fig. 2d and Supplementary Fig. 7). At the given resolution, there will be an ensemble of possible orientations for the fluorescent proteins with respect to each other, and only one structure is represented here. In the absence of calcium, Twitch-1 forms an elongated structure, with the two fluorescent proteins located at the respective ends. Upon calcium saturation, the protein shortens and becomes more compact, increasing the proximity of the fluorophores. The fits allow ambiguities about the precise distance between the fluorophores in the calcium-bound form, so we did not try to further relate the changes in geometry to changes in FRET. Overall, the shape changes detected here grossly resemble the shape changes reported for the biosensor $\mathrm{TN}-\mathrm{XXL}^{33}$. Thus, this analysis shows that a minimal TnC domain of 68 amino acids is sufficient to initiate a strong calcium-induced conformational change and can be useful for the construction of FRET biosensors. d
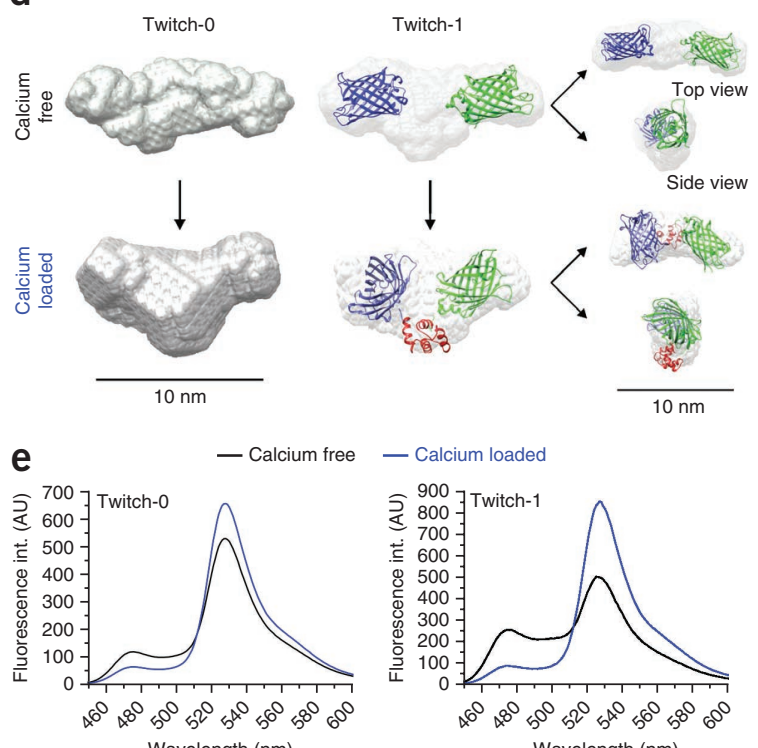

\section{Screening of diversified FRET calcium-sensor libraries}

We then created a large library of sensors with diversified linkers between the minimal calcium binding domain and the adjacent fluorophores (Fig. 1b and Supplementary Fig. 8). Additionally, we selected several residues as hotspots for mutagenesis by examining the solved NMR structure (Figs. $\mathbf{1} \mathbf{b}$ and $\mathbf{2 a}$ ), focusing on potential hinge regions where helices interact and nonchelating residues within the binding loops (Fig. 2 and Supplementary Fig. 8). To identify sensors with large responses, we implemented a twostep screening strategy (Fig. 3a). A functional bacterial colony prescreening assay was developed that allowed us to sample up to 1,000 colonies per plate, each expressing a diversified minimal domain sensor variant. As FRET sensors were not efficiently exported to the periplasmic space ${ }^{15}$, we developed a permeabilization protocol that allowed us to efficiently introduce calcium into the bacterial cytosol while collecting $R_{\min }$ and $R_{\max }$ values of the sensors (Online Methods). FRET changes were monitored by wide-field imaging with a charge-coupled device (CCD) camera (Supplementary Fig. 9). Overall, we screened about 100,000 bacterial colonies for optimal linker configurations and initially purified about 1,000 of those (Fig. 3a,b). We selected the bestperforming linker configurations and then performed additional hotspot mutagenesis within the minimal domain, thereby screening another 70,000 colonies and purifying another 1,000 proteins for further improvements in ratio change on a smaller scale (Fig. 3a,b). Notably, we were able to find minimal domain sensor variants that showed ratio changes of up to or more than $1,000 \%$, from no calcium to calcium saturation. We selected approximately 120 variants that showed the highest maximal ratio change as well as a small number of sensors with lower affinity and undertook a secondary screen in primary hippocampal neurons (Fig. 3c-e). Neurons expressing the variants were exposed to electrical field stimulation consisting of 1-160 individual pulses (Fig. 3c-e). Two variants showed strong improvements over TN-XXL and Twitch-1 in detecting subtle elevations in the cytosolic free 
a
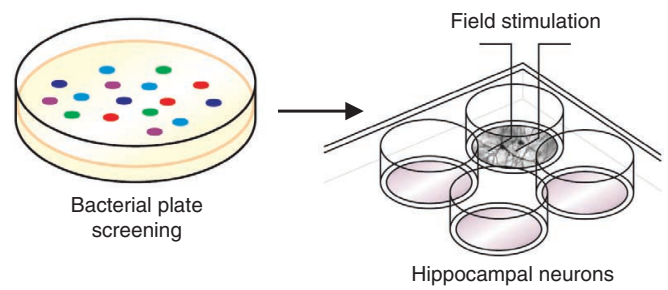

Hippocampal neurons b

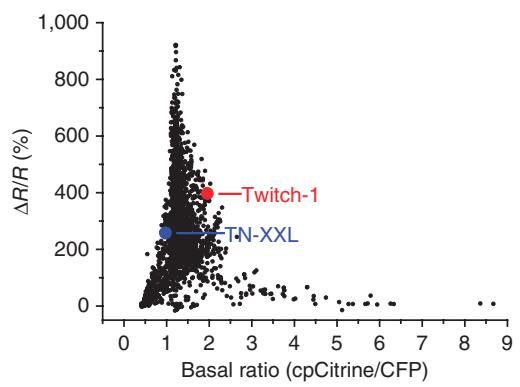

C
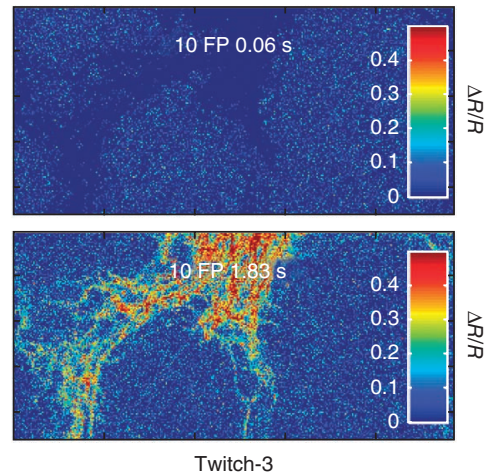

Twitch-3
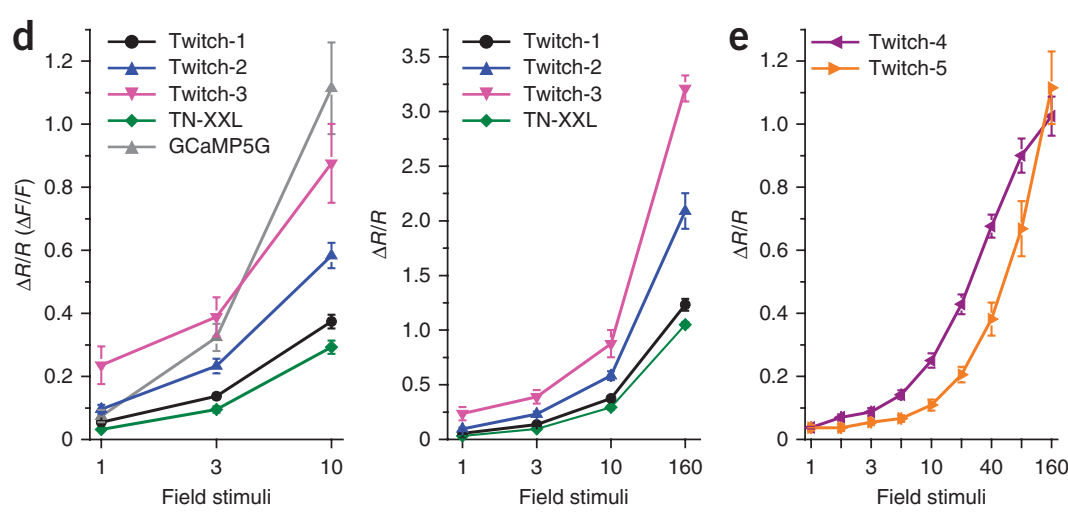

Figure 3 | Functional screening of large FRET calcium-sensor libraries. (a) Schema of the two-step screen consisting of a large-scale bacterial colony prescreening and a refining secondary screening in primary neurons used to select Twitch calcium-sensor variants. (b) Basal fluorescence ratios at zero calcium plotted versus calcium-induced ratio changes for $>2,000$ recombinant Twitch sensors. As a reference, the values for TN-XXL and the prototypical minimal domain sensor Twitch-1 are indicated. In smallscale purifications, low amounts of contaminating calcium are still present; thus the values for maximal calcium-induced ratio changes are slightly lower, and the values for basal starting ratios, slightly higher than when the proteins are purified stringently. (c) FRET ratio image of hippocampal neurons expressing Twitch-3 at onset (0.06 s) and after a field stimulus evoking ten action potentials $(1.83 \mathrm{~s})$. (d) Changes in fluorescence ratio of selected Twitch variants in response to short-field stimuli eliciting 1-160 action potentials. For comparison, responses of TN-XXL and GCaMP5G taken side by side are plotted. Error bars, mean \pm s.e.m. $(n=60-204)$. (e) Changes in fluorescence ratio of the low-affinity sensors Twitch-4 $(n=29)$ to Twitch-5 $(n=6)$ to 1-160 action potentials. Error bars, mean \pm s.e.m.

calcium concentration and were named Twitch-2 and Twitch-3 sensors (Fig. 3d). The maximal response of Twitch-2 and Twitch-3 was also improved compared to TN-XXL. We also found two sensors, Twitch-4 and Twitch-5, that showed lower affinity and sensitivity for calcium in the neurons, but that had faster response kinetics (Fig. 3e). These sensors may be useful for quantifying larger calcium fluxes in cells. In a separate set of neuronal screening experiments, Twitch-3 was compared to other known FRETbased calcium sensors (Supplementary Fig. 10). The core in vitro characteristics of selected Twitch indicators are summarized in Supplementary Table 3.

\section{Imaging neuronal activity in vivo with Twitch sensors}

To test the performance of Twitch sensors in vivo, we first expressed Twitch-3 in mouse primary visual cortex (V1) using adeno-associated virus (AAV) vector-mediated gene transfer ${ }^{11}$ and monitored calcium changes of neurons in V1 in response to presentation of moving gratings of differing orientations (Fig. 4a-e and Supplementary Figs. 11 and 12). We compared the in vivo properties of Twitch-3 to that of the established FRET based GECI YC3.60 (ref. 16). At comparable average laser powers Twitch-3 was brighter than YC3.60 (Supplementary Fig. 12). The total fraction of V1 neurons that responded to visual stimuli was larger in experiments usingTwitch-3 (34.8 $\pm 8.6 \%$, mean \pm weighted s.d.) than in those using YC3.60 (ref. 16) $(19.2 \pm 9.4 \%$, weighted s.d.), and the number of responding cells expressing Twitch-3 equaled the numbers of the synthetic fluorescent calcium indicator OGB-1 (36 $\pm 4 \%$, weighted s.d.) (Fig. 4e, note that OGB-1 data were acquired with slower acquisition rate and presumably higher signal-to-noise ratio; details in Online Methods).

New variants of cyan fluorescent protein such as mCerulean3 (ref. 34) or mTurquoise2 (ref. 35) have recently been generated. Both proteins have high quantum yields and improved photostability. We sought to improve the brightness of the Twitch sensors further by exchanging ECFP with these brighter donors and cpCitrine with cpVenus ${ }^{\mathrm{CD}}$ (ref. 36), a codon-diversified variant of the YFP variant Venus ${ }^{37}$. Although the proteins were almost identical, the exchange had a surprisingly negative impact on the FRET changes observed in the sensors (data not shown). We overcame this by screening additional libraries of extended linkers. In this way, we developed Twitch-2B, which retained the high FRET change of its precursor but was approximately twofold brighter in the donor emission channel than the previous Twitch sensors (Twitch-2B has cpVenus ${ }^{\mathrm{CD}}$ and mCerulean 3 as a FRET pair; Supplementary Fig. 13). Twitch-2B had a Hill coefficient of 1.3 and reported action potentials (APs) in cortical neurons in acute tissue slices in a fairly linear manner (Fig. $\mathbf{4 f , g}$ ). In this preparation, the mean change in fluorescence ratio $(\Delta R / R)$ in response to a single action potential stimulation was $26.5 \pm 3.8 \%$ (s.e.m., $n=11$; Fig. $\mathbf{4 g}$ ) and the increase in the amplitude of the signal with increasing numbers of action potentials was well approximated by a linear fit with the slope of $12.5 \%$ ratio change/AP.

We further investigated the properties of Twitch-2B and Twitch-3 in vivo by imaging sensory-evoked calcium signals in adult-born juxtaglomerular neurons of the mouse olfactory bulb $^{21}$ after lentivirus-mediated gene transfer. The amplitude of 

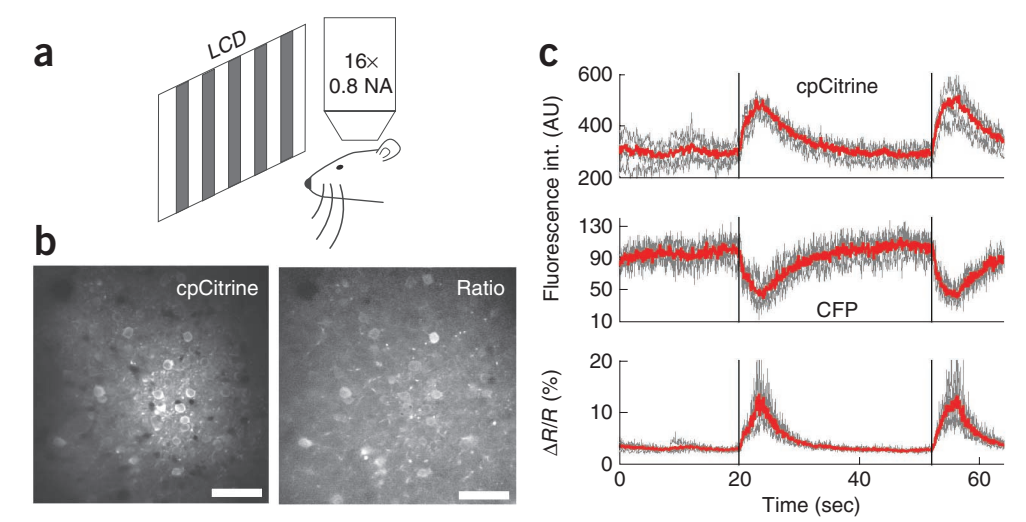

f Single trial

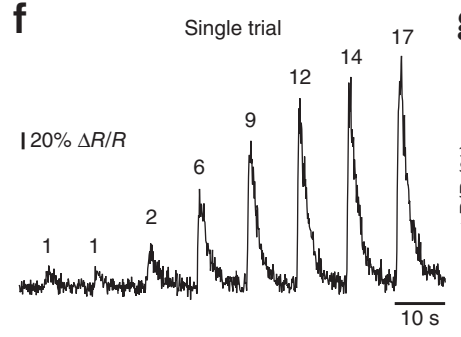

g
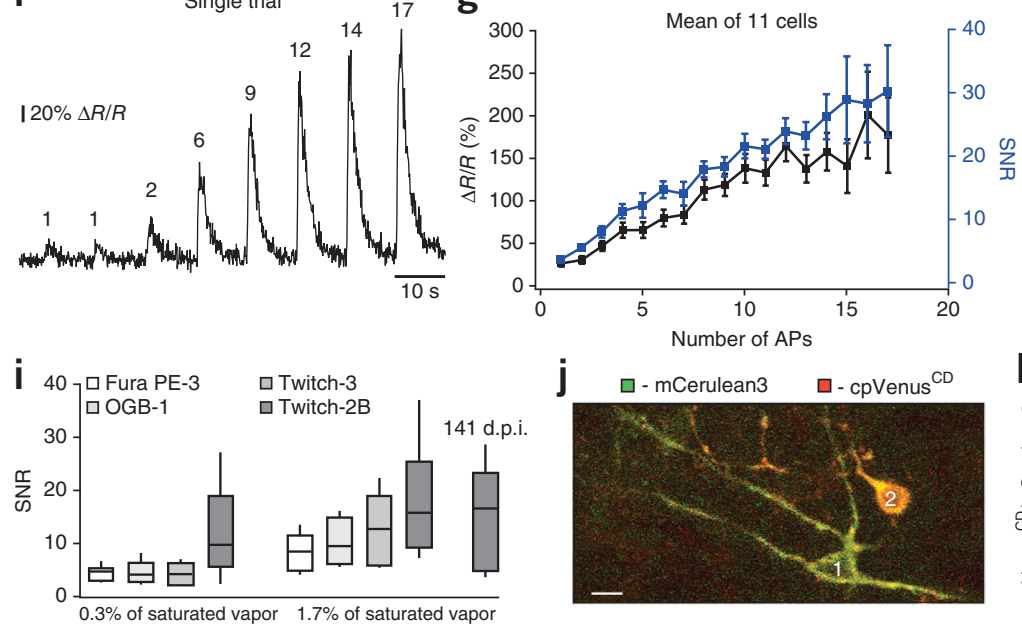
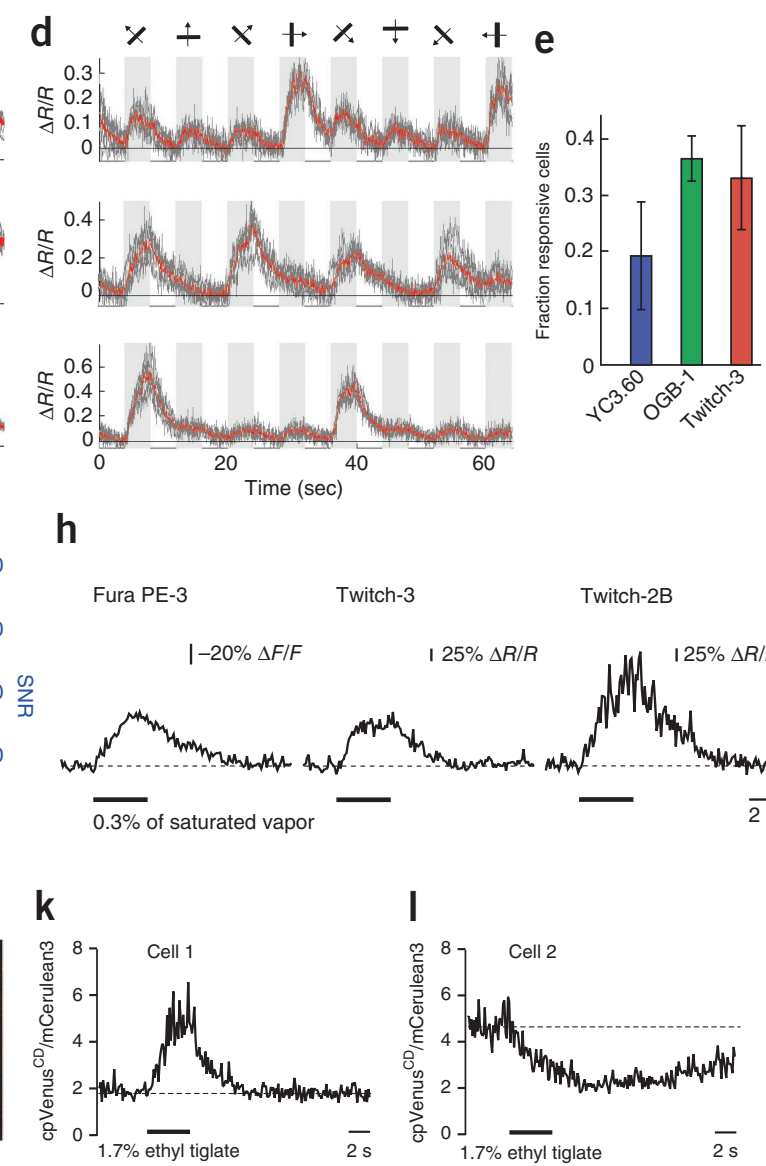

h

Fura PE-3

Twitch-3

Twitch-2B
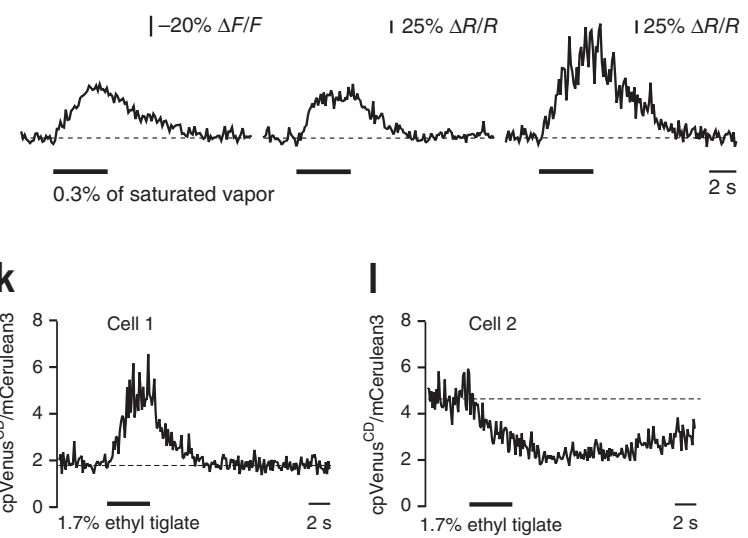

Figure 4 | Use of Twitch-3 and Twitch-2B for high-resolution in vivo imaging in the mouse brain. (a) Schematic showing experimental setup for recording visually evoked calcium signals. (b) Twitch-3 cpCitrine fluorescence in layer 2/3 neurons 4 weeks after AAV injection (left) and ratio cpCitrine/CFP image (right). Scale bars, $50 \mu \mathrm{m}$. (c) Measured cpCitrine (top) and CFP (middle) channels for five repetitions of visual stimuli consisting of moving gratings. Bottom, the ratio between these two channels. Gray, single trials; red, trial average; vertical lines show the preferred stimulus start time. (d) Responses of three cells to eight different moving grating stimuli. (e) Fraction of visually responsive neurons in in vivo experiments when using three different calcium indicators (OGB-1, $36 \pm 4 \%$; YC3.60, $19.2 \pm 9.4 \%$; Twitch-3, $34.8 \pm 8.6 \%$; mean \pm weighted s.d.; neuropil compensation factor $=0.7$, data for OGB-1 from ref. 11). Significances were determined according to Mann-Whitney test. YC3.60, Twitch-3 $P=0.052 ; 0$ GB-1, YC3.60 $P=0.143 ; 0$ GB-1, Twitch-3 $P=0.548$; data for YC3.60, Twitch-3 and OGB-1 are from five, six and three animals, respectively (Online Methods). (f) Single trial example (of a total of 11 cells) of the relationship between the emission-ratio change of Twitch-2B and the number of underlying action potentials recorded from a layer $2 / 3$ pyramidal neuron in a cortical slice at $35^{\circ} \mathrm{C}$. (g) Relationship between the mean amplitude of Twitch-2B-mediated calcium transients as well as mean signal-to-noise ratio (SNR, see Online Methods) and the number of underlying action potentials in a cortical slice $(n=11$, data from four animals, mean \pm s.e.m.). (h) Representative (from 15, 7 and 12 cells, respectively) sensory-evoked calcium transients in juxtaglomerular neurons of the in vivo olfactory bulb recorded with three different calcium sensors, as indicated. At this low concentration, odorants evoke calcium transients in cells labeled both with synthetic (for example, Fura PE-3) and with genetically encoded (Twitch-2B and Twitch-3) indicators. (i) Representative box plots showing SNRs measured in vivo in olfactory bulb neurons labeled with four different indicators in response to two odorant concentrations, as indicated (from left to right, $n=15,38,7,12,15,32,9,15,11$ cells, respectively; data from ten mice). For all data sets involving GECIs, recordings were conducted at 35-37 d after infection (d.p.i.). The last box plot shows data obtained at $141 \mathrm{~d}$.p.i. from one mouse also imaged at 35-37 d.p.i. The significances for differences in SNR values were determined according to Mann-Whitney test (for 0.3\% saturated vapor, Twitch-2B, Fura PE-3 $P=0.005 ;$ Twitch-2B, 0GB-1 $P=0.0016$; Twitch-2B, Twitch-3 $P=0.015$; for $1.7 \%$ saturated vapor, Twitch-2B, Fura PE-3 $P=0.003$; Twitch-2B, 0GB-1 $P=0.0045 ;$ Twitch-2B, Twitch-3 $P=0.156$; Twitch-2B, Twitch-2B 141 d.p.i. $P=0.54) . P>0.05$ for all other possible comparisons. Boxes drawn from 75 th percentile (top) to 25 th percentile (bottom); whiskers, 85th percentile to 10th percentile. (j) Image of two adult-born juxtaglomerular neurons taken $37 \mathrm{~d}$ after the injection of lentiviral vectors encoding Twitch-2B. Scale bar, $10 \mu \mathrm{m} .(\mathbf{k}, \mathbf{l})$ On application of the odorant (ethyl tiglate), cell 1 (k) shows an increased calcium response, whereas cell 2 (l) shows a decreased response.

Twitch-3 signals was comparable to that of signals reported by the synthetic calcium indicator dye Fura PE-3 (Fig. 4h), whereas Twitch-2B signals were stronger than those of both Twitch-3 and Fura PE-3. Signal-to-noise ratios were calculated for Twitch-2B and Twitch-3 as well as Fura PE-3 and OGB-1 in the labeled neurons and in response to two different odorant concentrations (Fig. 4i). In both conditions, Twitch-2B showed higher signal-tonoise ratios than synthetic indicators whereas the signal-to-noise ratios obtained using Twitch-3 were comparable to those of Fura PE-3 and OGB-1.

One of the main advantages of Twitch sensors compared to other GECIs is their potential for use in long-term imaging experiments. To assess this, we compared the responses of Twitch-2B in adult-born olfactory neurons to the same odorant stimuli at two different time points in vivo. We observed that the quality of Twitch-2B-mediated responses did not change over time 
(Fig.4i), suggesting that this indicator is well suited for longterm chronic imaging (Fig. 4i). In addition, we looked for signs of toxicity in these mice, and at $141 \mathrm{~d}$ after infection, we found that only 2 out of 63 juxtaglomerular neurons analyzed had filled nuclei, a potential morphological indication of unhealthy cells. In addition, owing to its high dynamic range, Twitch-2B allowed visualization of neurons with different levels of basal activity (Fig. 4j-1). Out of two cells shown in Figure 4j, cell 1 shows low
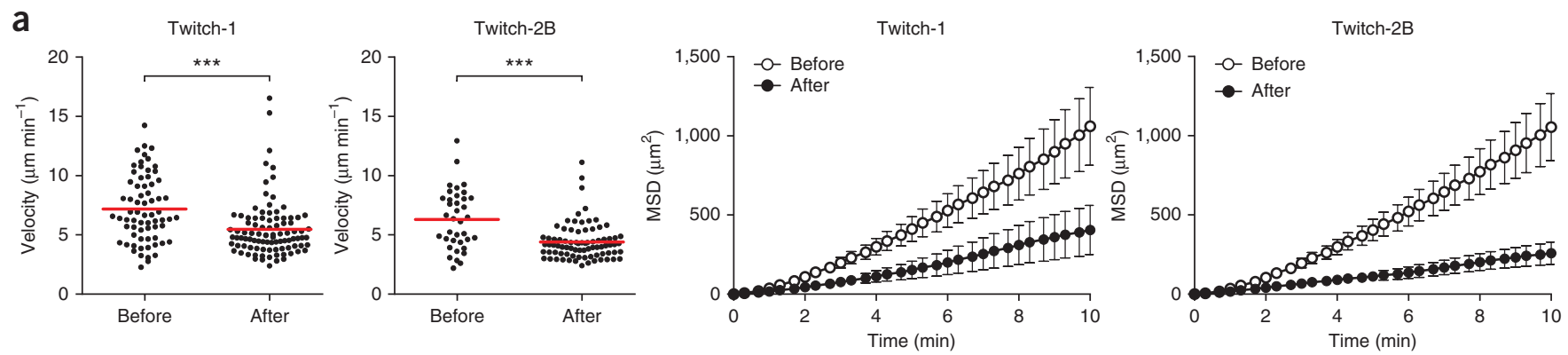

b
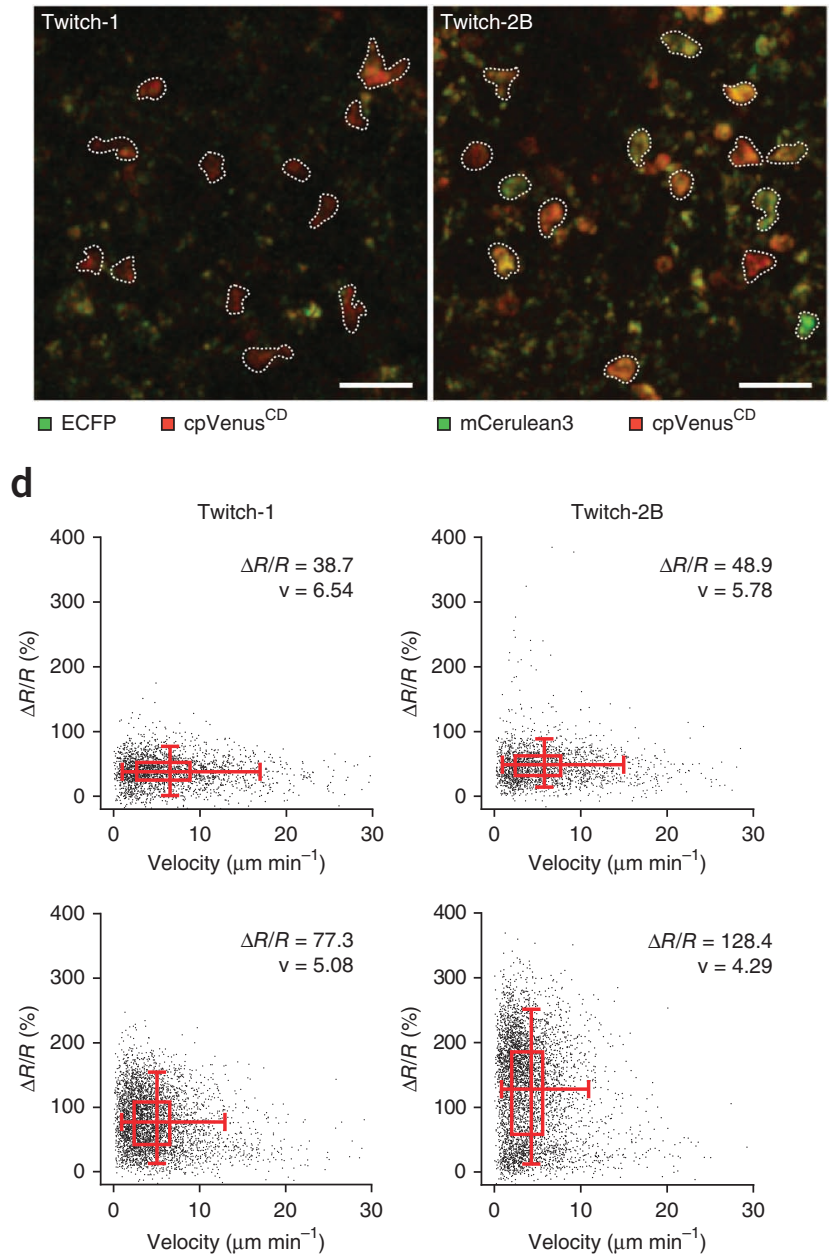

C $\square$ ECFP $\square$ cpVenus $^{\mathrm{CD}}$
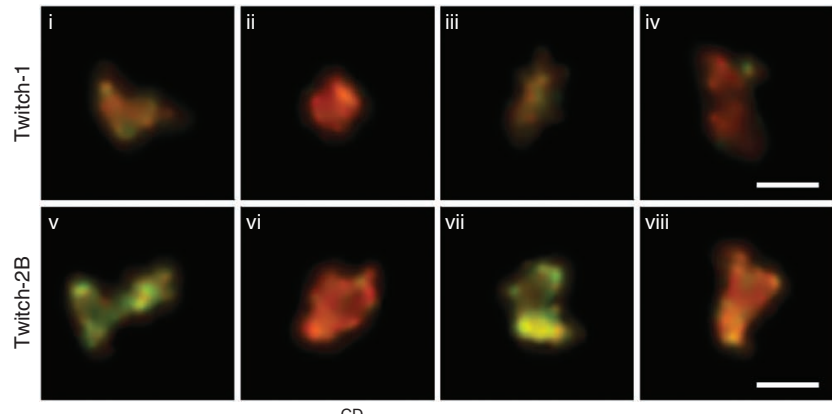

$\square$ mCerulean3

$$
\text { e }
$$
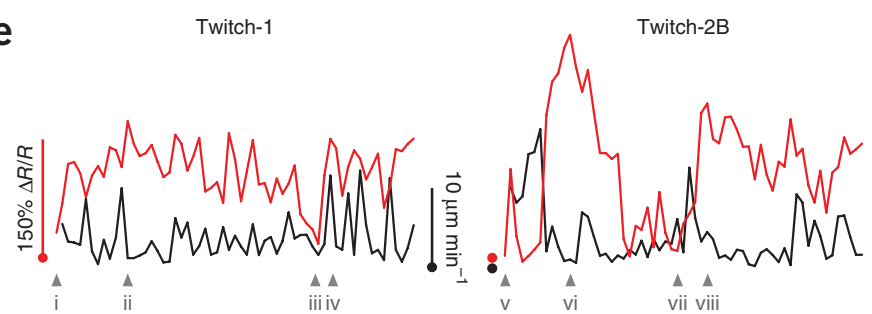

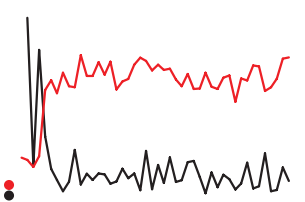

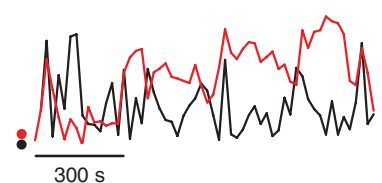

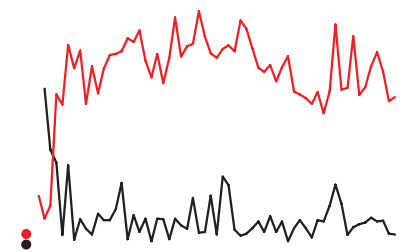

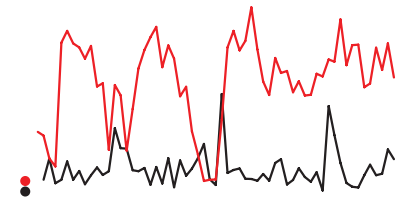

Figure 5 | In vivo calcium imaging of T lymphocytes with Twitch-1 and Twitch-2B. (a) Quantification of T lymphocyte locomotion within the popliteal lymph node before and after i.v. injection of $100 \mu \mathrm{g}$ of OVA peptide antigen. Average velocities and the mean squared displacement (m.s.d. \pm s.e.m.) for Twitch-1- and Twitch-2B-expressing T lymphocytes. $n=68$ and 94 cells (Twitch-1) and 37 and 80 cells (Twitch-2B) for control and antigensupplemented conditions, respectively. For every condition, data were obtained from three animals. ${ }^{* *} P<0.0001$ (Mann-Whitney $U$ test). Red lines in the left two graphs indicate mean values. (b) Sample images from in vivo calcium imaging of OT-II T lymphocytes in the popliteal lymph node after antigen injection. Overlay of donor and acceptor fluorescence shown. Scale bars, $30 \mu \mathrm{m}$. (c) Selected time-lapse series of individual cells expressing Twitch-1 or Twitch-2B, oscillating through minimal and maximal $\Delta R / R$. Numbers indicate time points in e. Scale bars, $10 \mu \mathrm{m}$. Data are representative traces of experiments done in three animals. (d) Scatter plots of cell velocity versus calcium-indicator ratio change for each time point analyzed before (top) and after (bottom) antigen injection. Mean values for $\Delta R / R$ and velocity $(v)$ are indicated along with a two-dimensional box plot. Box plot indicates the 25th and 75th percentiles, and the whiskers represent the 5th and 95th percentiles. $P<0.001$. (e) Sample tracks of intracellular calcium levels (red) and T cell velocities (black) after the application of antigen. Top, the two cells from c. Dots in e depict zero levels for ratio (red) and velocity (black). Results from three animals before and after antigen exposure are shown. 
cpVenus $\mathrm{CD} / \mathrm{mCerulean} 3$ ratio (1.9), whereas cell 2 shows high cpVenus $\mathrm{CD} / \mathrm{mCerulean} 3$ ratio (4.6), presumably because of the high basal firing rate of the neuron ${ }^{21}$. In response to the same sensory stimulus, cell 1 responded with an increase in calcium signal (Fig. 4k), and cell 2 responded with a decrease (Fig. 4l) in the intracellular free calcium concentration. Cells with decreased responses were encountered five times during this study. In total, such cells comprise $12 \%$ of the odor-responsive population of juxtaglomerular neurons ${ }^{21}$.

\section{Imaging T lymphocyte activation in vivo using Twitch-2B}

Autoreactive T lymphocytes cause autoimmune diseases and are highly motile cells. As such, they offer an ideal platform to test the usefulness of ratiometric calcium indicators for functional imaging in moving samples ${ }^{38}$. Because $\mathrm{T}$ cells are highly motile and quickly change their location within the inhomogeneous tissue environment, their brightness will continuously vary over time. To normalize for this effect, only ratiometric calcium indicators are suitable. We previously worked out parameters to express FRET calcium indicators in autoantigen-specific $\mathrm{T}$ cells and protocols to image them ${ }^{36}$. To test the performance of the advanced Twitch variants, we imaged immune cells during antigen recognition in vivo. Antigen recognition by T cells triggers a swift rise of the intracellular free calcium concentration, which can be used as an indication of $\mathrm{T}$ cell activation ${ }^{39}$. We transduced antigenspecific T cells from OT-II mice ${ }^{40}$ with retroviruses expressing Twitch-1 or Twitch-2B and compared the antigen-dependent activation of these T cells by dendritic cells (DC) in in vivo mouse lymph nodes. In the absence of antigen, both cell lines showed comparable motility and displacement and had low intracellular free $\mathrm{Ca}^{2+}$ concentration (Fig. 5a,d). After intravenous (i.v.) injection of the cognate OVA peptide antigen, both cell lines reduced their motility and displayed elevated calcium levels (Fig. 5a,b,d). Cells expressing Twitch-2B displayed a ratio change of $>300 \%$ in vivo and showed a higher dynamic range of the sensor output than cells expressing Twitch-1 (Fig. 5b,d). The cells expressing Twitch-2B showed clear fluctuations from green to yellow fluorescence, allowing visual estimation of changes in calcium level without the need for pseudocoloring (Fig. 5b,c). The increased dynamic range of Twitch-2B improved the resolution of the calcium oscillation events in T cells (Fig. $\mathbf{5 c}, \mathbf{e})$.

\section{DISCUSSION}

Here we describe a series of optimized FRET-based calcium indicators for ratiometric in vivo imaging. We based our work on previous sensors based on $\mathrm{TnC}$ - the calcium sensor from skeletal and cardiac muscle-as calcium-binding moiety ${ }^{6,20}$. We illustrate the usefulness of optimized Twitch variants by monitoring neuronal activity and studying activation patterns of $\mathrm{T}$ lymphocytes in vivo with high sensitivity. This approach may be easily extended to other motile cell types such as B lymphocytes or thrombocytes and could become very valuable for studying the interactions that these cells encounter as they move through tissue microenvironments within the body.

We identified the C-terminal lobe domain of $\mathrm{TnC}$ as the smallest possible domain for generating GECIs that exhibits a large conformational change upon calcium binding. Sensors based on this domain are smaller, can lead to reduced sensor-induced buffering during long-term chronic expression and minimize the risk of cytotoxicity. Reducing the size of the calcium-binding domain may also help to simplify response properties compared to other sensors. In vitro titrations of Twitch-2B, for example, showed improved Hill coefficient values compared, for example, to recent G-CaMP5 variants ${ }^{11}$. As with other GECIs, a trade-off can be seen in Twitch sensors between high-affinity binding and fast response kinetics. Whereas sensors with somewhat lower affinity such as Twitch-5 $\left(K_{\mathrm{d}}=9.25 \mu \mathrm{M}, \tau=0.16 \mathrm{~s}\right)$ show relatively fast kinetics, the most sensitive variants, such as Twitch-3 ( $\left.K_{\mathrm{d}}=250 \mathrm{nM}, \tau=1.5 \mathrm{~s}\right)$, are relatively slow. Although slow kinetics may increase sensitivity, as more signal photons are generated, sensitive indicators with brisker responses are also required and will need to be developed.

Finally, to optimize the FRET change of the sensors, we developed a strategy for sensor prescreening in bacterial colonies that allowed us to assay a relatively large number of modified sensors and identify interesting candidates for further analysis. In this way we improved the overall FRET change of Twitch sensors upon calcium binding, from $10-20 \%$ ratio change initially to $>1,000 \%$. Similar screening techniques might be useful to improve some of the existing FRET biosensors for other signal molecules, many of which currently show modest maximal ratio changes.

\section{METHODS}

Methods and any associated references are available in the online version of the paper.

Accession codes. Biological Magnetic Resonance Data Bank: chemical shift assignments are available under accession number 19285. Protein Data Bank: the final atomic coordinates are available under accession number $2 \mathrm{~m} 97$. Addgene: Twitch-2B (in pcDNA3) is available under accession number 48203. GenBank: the Twitch-2B sequence has been deposited under the accession number KF843821.

Note: Any Supplementary Information and Source Data files are available in the online version of the paper.

\section{ACKNOWLEDGMENTS}

This work was funded by the Max Planck Society, the Howard Hughes Medical Institute, Deutsche Forschungsgemeinschaft (DFG) grant SFB 870 (to 0. Griesbeck), DFG grant GRK1721 (to G.W.), EU FP7 EuroV1sion grant (to 0. Griesbeck), US National Science Foundation grant IBN-9985315 (to T.A.), US National Science Foundation grant IOS 1145981 (to L.C.R.) and the DFG Center for Integrative Neuroscience (to 0. Garaschuk).

\section{AUTHOR CONTRIBUTIONS}

T.T. characterized the minimal domain, cloned constructs and performed protein purifications and in vitro spectroscopic characterizations; J.L. established the bacterial colony screen and performed colony screening and further protein purifications; M.M. and I.B. performed in vivo imaging of T lymphocytes; L.R., S.B., Y. Laukat and C.G. performed NMR structure determination and interpreted results; T.A. and L.C.R. cloned toadfish TnC; A.G. and T.T. collected SAXS data; G.W. calculated SAXS models; H.D. performed in vivo characterization in mouse visual cortex; Y.K., Y. Liang, G.K. and 0. Garaschuk planned, performed and interpreted characterization of the sensors in cortical slices in situ and mouse olfactory bulb in vivo; T.T., T.W.C., H.D. and D.S.K. planned, performed and interpreted neuronal screening results. 0 . Griesbeck designed experiments, supervised sensor engineering and screening and integrated results from the collaborators. T.T., H.D., C.G., 0. Garaschuk and 0 . Griesbeck wrote the manuscript.

\section{COMPETING FINANCIAL INTERESTS}

The authors declare no competing financial interests. 
Reprints and permissions information is available online at http://www.nature. com/reprints/index.html.

1. Palmer, A.E., Quin, Y., Park, J.G. \& McCombs, J.E. Design and application of genetically encoded biosensors. Trends Biotechnol. 29, 144-152 (2011).

2. Looger, L.L. \& Griesbeck, 0. Genetically encoded neural activity indicators. Curr. Opin. Neurobiol. 22, 18-23 (2012).

3. Knöpfel, T. Genetically encoded optical indicators for the analysis of neuronal circuits. Nat. Rev. Neurosci. 13, 687-700 (2012).

4. Mank, M. \& Griesbeck, 0. Genetically encoded calcium indicators. Chem. Rev. 108, 1550-1564 (2008).

5. Miyawaki, A. et al. Fluorescent indicators for $\mathrm{Ca}^{2+}$ based on green fluorescent proteins and calmodulin. Nature 388, 882-887 (1997).

6. Heim, N. \& Griesbeck, 0. Genetically encoded indicators of cellular calcium dynamics based on troponin $\mathrm{C}$ and green fluorescent protein. J. Biol. Chem. 279, 14280-14286 (2004).

7. Wang, Q., Shui, B., Kotlikoff, M.I. \& Sondermann, H. Structural basis for calcium sensing by GCaMP2. Structure 16, 1817-1827 (2008).

8. Akerboom, J. et al. Crystal structures of the GCaMP calcium sensor reveal the mechanism of fluorescence signal change and aid rational design. J. Biol. Chem. 284, 6455-6464 (2009).

9. Nakai, J., Ohkura, M. \& Imoto, K. A high signal-to-noise $\mathrm{Ca}^{2+}$ probe composed of a single green fluorescent protein. Nat. Biotechnol. 19, 137-141 (2001).

10. Tian, L. et al. Imaging neural activity in worms, flies and mice with improved GCaMP calcium indicators. Nat. Methods 6, 875-881 (2009).

11. Akerboom, J. et al. Optimization of a GCaMP calcium indicator for neural activity imaging. J. Neurosci. 32, 13819-13840 (2012).

12. Zariwala, H.A. et al. A Cre-cependent GCaMP3 reporter mouse for neuronal imaging in vivo. J. Neurosci. 32, 3131-3141 (2012).

13. Ohkura, M. et al. Genetically encoded green fluorescent $\mathrm{Ca}^{2+}$ indicators with improved detectability for neuronal $\mathrm{Ca}^{2+}$ signals. PLOS ONE 7, e51286 (2012).

14. Akerboom, J. et al. Genetically encoded calcium indicators for multi-color neural activity imaging and combination with optogenetics. Front. Mol. Neurosci. 6, 2 (2013).

15. Zhao, Y. et al. An expanded palette of genetically encoded $\mathrm{Ca}^{2+}$ indicators. Science 333, 1888-1891 (2011).

16. Nagai, T., Yamada, S., Tominaga, T., Ichikawa, M. \& Miyawaki, A. Expanded dynamic range of fluorescent indicators for $\mathrm{Ca}^{2+}$ by circularly permuted yellow fluorescent proteins. Proc. Natl. Acad. Sci. USA 101, 10554-10559 (2004).

17. Lütcke, H. et al. Optical recording of neuronal activity with a geneticallyencoded calcium indicator in anesthetized and freely moving mice. Front. Neural Circuits 4, 9 (2010).

18. Palmer, A.E. et al. $\mathrm{Ca}^{2+}$ indicators based on computationally redesigned calmodulin-peptide pairs. Chem. Biol. 13, 521-530 (2006).

19. Horikawa, K. et al. Spontaneous network activity visualized by ultrasensitive $\mathrm{Ca}^{2+}$ indicators, yellow Cameleon-Nano. Nat. Methods 7 , 729-732 (2010).

20. Mank, M. et al. A genetically encoded calcium indicator for chronic in vivo two-photon imaging. Nat. Methods 5, 805-811 (2008).
21. Homma, R. et al. In vivo functional properties of juxtaglomerular neurons in the mouse olfactory bulb. Front. Neural Circuits 7, 23 (2013).

22. Kuchibhotla, K.V. et al. $A \beta$ plaques lead to aberrant regulation of calcium homeostasis in vivo resulting in structural and functional disruption of neuronal networks. Neuron 59, 214-225 (2008).

23. Siffrin, V. et al. In vivo imaging of partially reversible Th17 cell-induced neuronal dysfunction in the course of encephalomyelitis. Immunity $\mathbf{3 3}$, 424-436 (2010).

24. Vassylyev, D.G. et al. Crystal structure of troponin C in complex with troponin I fragment at 2.3-angstrom resolution. Proc. Natl. Acad. Sci. USA 95, 4847-4852 (1998).

25. Gordon, A.M., Homsher, E. \& Regnier, M. Regulation of contraction in striated muscle. Physiol. Rev. 80, 853-924 (2000).

26. Direnberger, S. et al. Biocompatibility of a genetically encoded calcium indicator in a transgenic mouse model. Nat. Commun. 3, 1031 (2012).

27. DeMaria, C.D., Soong, T.W., Alseikhan, B.A., Alvania, R.S. \& Yue, D.T. Calmodulin bifurcates the local $\mathrm{Ca}^{2+}$ signal that modulates $\mathrm{P} / \mathrm{Q}$-type $\mathrm{Ca}^{2+}$ channels. Nature 411, 484-489 (2001).

28. Mank, M. et al. A FRET-based calcium biosensor with fast signal kinetics and high fluorescence change. Biophys. J. 90, 1790-1796 (2006).

29. Wishart, D.S. \& Case, D.A. Use of chemical shifts in macromolecular structure determination. Methods Enzymol. 338, 3-34 (2001).

30. Slupsky, C.M. \& Sykes, B.D. NMR solution structure of calcium-saturated skeletal muscle troponin C. Biochemistry 34, 15953-15964 (1995).

31. Huang, J.R. \& Grzesiek, S. Ensemble calculations of unstructured proteins constrained by RDC and PRE data: a case study of urea-denatured ubiquitin. J. Am. Chem. Soc. 132, 694-705 (2010).

32. Petoukhov, M.V. et al. New developments in the ATSAS program package for small-angle scattering data analysis. J. Appl. Cryst. 45, 342-350 (2012).

33. Geiger, A. et al. Correlating calcium binding, Förster resonance energy transfer, and conformational change in the biosensor TN-XXL. Biophys. J. 102, 2401-2410 (2012).

34. Markwardt, M.L. et al. An improved cerulean fluorescent protein with enhanced brightness and reduced reversible photoswitching. PLOS ONE 6 , e17896 (2011).

35. Goedhart, J. et al. Bright cyan fluorescent protein variants identified by fluorescence lifetime screening. Nat. Methods 7, 137-139 (2010).

36. Mues, M. et al. Real-time in vivo analysis of T cell activation in the central nervous system using a genetically encoded calcium indicator. Nat. Med. 19, 778-783 (2013).

37. Nagai, T. et al. A variant of yellow fluorescent protein with fast and efficient maturation for cell-biological applications. Nat. Biotechnol. 20, 87-90 (2002)

38. Bousso, P. \& Moreau, H.D. Functional immunoimaging: the revolution continues. Nat. Rev. Immunol. 12, 858-864 (2012).

39. Feske, S., Skolnik, E.Y. \& Prakriya, M. Ion channels and transporters in lymphocyte function and immunity. Nat. Rev. Immunol. 12, 532-547 (2012).

40. Barnden, M.J. et al. Defective TCR expression in transgenic mice constructed using cDNA-based $\alpha$ - and $\beta$-chain genes under the control of heterologous regulatory elements. Immunol. Cell Biol. 76, 34-40 (1998). 


\section{ONLINE METHODS}

Gene construction and biosensor library design. cDNA of toadfish troponin $\mathrm{C}$ (ts $\mathrm{TnC}$ ) retrieved from white muscle and swim bladder was used as starting material for designing the new minimal domain calcium indicators. To generate the Twitch single C-terminal domain the region from amino acid Ser93 to Gln161 of ts TnC was amplified and cloned in between the FRET fluorescent protein variants. Point mutations were introduced by site-directed mutagenesis using the primer extension method similar to the QuickChange method (Stratagene) and by errorprone PCR (Jena Error-Prone Kit). Proline and random linkers were introduced via PCR using primer extensions fused to ts $T n C$ at the $5^{\prime}$ and $3^{\prime}$ ends of the C-terminal domain. The general structure of the calcium biosensors described herein is as follows: ECFP/Cer3/mTurquoise2-SphI-linker-minimal calcium binding domain-linker-SacI-Met cpCitrine174/cpVenus ${ }^{\mathrm{CD}}$-Stop (Fig. 1). For specific point mutations and linkers of Twitch variants, see Supplementary Figure 8 and Supplementary Table 3. Constructs encoding Twitch calcium sensors can be viewed at http://www. addgene.org/Oliver_Griesbeck/

Cloning of toadfish troponin C. Total RNA was isolated from $0.1 \mathrm{~g}$ toadfish swim bladder muscle following homogenization in presence of $0.9 \mathrm{ml}$ TRIzol in accordance with accompanying instructions (Invitrogen, California). From an aliquot of total RNA, a lambda gt11 cDNA library was constructed with the CapSwitch Kit (ClonTech, California). The library was amplified once, and aliquots of the amplified library were screened by PCR for $\mathrm{TnC}$ sequences. The same was done for construction of a toadfish white muscle cDNA library.

For screening, a degenerate primer for $\mathrm{TnC}$ was paired with a primer specific to the lambda vector. The degenerate primer was designed with the help of the Blocks and CODEHOP algorithms (blocks.fhcrc.org/blocks/codehop.html), and the oligonucleotide recognized a distinct, highly conserved motif of the TnC protein: 5'-CGACTTCGACGAGTTCCTGGTNATGATGGT-3', recognizing the motif DFEEFLVMM at the start of helix D, after the second calcium-binding loop in the N-terminal half of TnC. Sequence information obtained from this amplification allowed design of primers specific for toadfish TnC. These primers were subsequently paired with lambda-specific primers to amplify the remainder of the full-length cDNA. As a verification of the sequence data, fulllength cDNA was then amplified from total RNA following reverse transcription, and the amplified product was sequenced.

NMR spectroscopy. The expression of the uniformly ${ }^{15} \mathrm{~N}$ - and ${ }^{13} \mathrm{C}$-labeled histidine-tagged minimal C-terminal lobe domain of Opsanus TnC (tsTnC), comprising EF hands 3 and 4, was performed at $37{ }^{\circ} \mathrm{C}$ in minimal medium with ${ }^{15} \mathrm{~N}$-ammonium chloride and ${ }^{13} \mathrm{C}_{6}$-d-glucose as the sole nitrogen and carbon sources. The fusion protein was purified by immobilized metal affinity chromatography on Ni-NTA agarose (Qiagen, Venlo, The Netherlands) and subsequently cleaved with TEV protease. The released tsTnC was further purified by reverse-phase HPLC.
NMR samples for structure determination of tsTnC were prepared at a final concentration of $1 \mathrm{mM}$ in $20 \mathrm{mM}$ Bis-Tris (pH 7.0), $100 \mathrm{mM} \mathrm{KCl}, 10 \mathrm{mM} \mathrm{DTT}, 10 \mathrm{mM} \mathrm{CaCl}_{2}$ and $10 \%{ }^{2} \mathrm{H}_{2} \mathrm{O}$. The calcium-free sample in presence of magnesium contained $10 \mu \mathrm{M}$ tsTnC, $100 \mu \mathrm{M}$ EGTA and $1 \mathrm{mM} \mathrm{MgCl}_{2}$.

NMR experiments were carried out at $303 \mathrm{~K}$ on a $700-\mathrm{MHz}$ Bruker DRX spectrometer equipped with a $z$-gradient cryoprobe and $900-\mathrm{MHz}$ spectrometer with a triple resonance $5-\mathrm{mm}$ cryoprobe with a $z$-axis pulsed field gradient. Triple resonance NMR experiments including 3D HNCA (refs. 41,42), 3D CBCANH (ref. 43) and 3D CBCA(CO)NH (ref. 42) were collected to obtain sequence-specific backbone and $\mathrm{C} \beta$ resonances assignment. Vicinal (three-bond) $\mathrm{H}_{\mathrm{N}}$ - $\mathrm{H} \alpha$ coupling constants $\left({ }^{3} \mathrm{JH}_{\mathrm{N}} \mathrm{H} \alpha\right.$ ) were evaluated from cross-peak intensities in quantitative J-correlation (HNHA) spectra ${ }^{44}$. Residual dipolar couplings ${ }^{1} \mathrm{D}_{\mathrm{NH}} \mathrm{RDC}$ were measured by taking the difference in the one-bond ${ }^{1} \mathrm{H}-{ }^{15} \mathrm{~N}$ splittings $\left({ }^{1} \mathrm{~J}_{\mathrm{NH}}+{ }^{1} \mathrm{D}_{\mathrm{NH}}\right)$ in aligned $(\sim 20 \mathrm{mg} / \mathrm{ml})$ phage pf1 (ref. 45$)$ and isotropic media using an in-phase/anti-phase (IPAP) HSQC experiment ${ }^{46}$.The spectra were processed using NMRpipe ${ }^{47}$ and analyzed by SPARKY (http://www.cgl.ucsf.edu/home/sparky/) and $\mathrm{CARA}^{48} \cdot{ }^{1} \mathrm{H},{ }^{13} \mathrm{C}$ and ${ }^{15} \mathrm{~N}$ chemical shifts were calibrated indirectly by external DSS references.

The structure calculation was performed with CS-RDCRosetta ${ }^{49,50}$ using as structural restraints the residual dipolar couplings, torsion angles $\phi / \psi$ derived from TALOS + database and the complete backbone and $\mathrm{C} \beta$ chemical shifts of those residues indicated by TALOS + to be rigid in picosecond timescale with an order parameter $\mathrm{S}^{2}>0.7$. A set of 200 fragment candidates matching these chemical shifts was used to calculate 3,000 structures in Rosetta. The energy of these Rosetta structures was then rescored against the observed chemical shifts and the 20 conformers with the lowest rescored energy were selected for the ensemble. The structures were visualized and evaluated using PyMOL (http:// www.pymol.org/), MOLMOL ${ }^{51}$, CHIMERA ${ }^{52}$, PROCHECK$\mathrm{NMR}^{53}$ and MolProbity ${ }^{54}$. The chemical shift assignments are available from the Biological Magnetic Resonance Data Bank (accession number 19285), and the final atomic coordinates are available from the Protein Data Bank (2m97). The distance between the $\mathrm{N}$ and $\mathrm{C}$ terminus was measured to be $14.7 \AA$ for the calcium-loaded globular conformation. For the calciumfree form, average distances between $\mathrm{N}$ and $\mathrm{C}$ terminus of $\sim 50 \AA$ were extrapolated from urea-disordered ubiquitin, which has a similar amino acid number as $\mathrm{TnC}^{31}$.

The GFP molecule is $\sim 4 \mathrm{~nm}$ in length and $\sim 2 \mathrm{~nm}$ wide. Depending on how the two $\beta$-barrels encounter each other, $\mathrm{d}^{\text {chromophore }}$ is $\sim 2-4 \mathrm{~nm}$. The Förster radius is normally around $R_{0}=5 \mathrm{~nm}$ for GFP variants. Thus, in the calcium-free form, a distance of $\left(5.2+\mathrm{d}^{\text {chromophore }}+5\right) \mathrm{nm}$ would be expected for the FRET effect, and in the calcium-loaded form $\left(1.5+\mathrm{d}^{\text {chromophore }}+5\right) \mathrm{nm}$ is expected. This distance change provides a ratio of $4-6$ between the FRET effects, which agrees reasonably well with the observed FRET changes for Twitch-1. Owing to the $\mathrm{r}^{-6}$ averaging of FRET, only the closest $20 \%$ of the conformations are taken into account:

$$
\frac{\text { FRET folded }}{\text { FRET unfolded }}=\frac{(\text { dchromophore }+d T n C \text { folded }+R 0)^{-6}}{p \text { TnCunfolded closest } 20 \%(\text { dchromophore }+d T n C \text { unfolded } 20 \%+R 0)^{-6}}
$$


SAXS. Small-angle-scattering data were collected on the ID14-3 beamline of the European Synchrotron Radiation Facility ESRF (Grenoble, France). Twitch samples were purified by size exclusion chromatography in buffer A (30 mM MOPS, $100 \mathrm{mM} \mathrm{KCl,}$ $100 \mathrm{mM}$ EDTA and $100 \mathrm{mM}$ EGTA, pH 7.5) then taken up either in buffer containing EGTA ( $2 \mathrm{mM}, \mathrm{Ca}^{2+}$ free) or $10 \mathrm{mM} \mathrm{CaCl}$ (10 $\mathrm{mM}$ high $\mathrm{Ca}^{2+}$ ) and centrifuged before the SAXS measurements. Samples were measured at concentrations of 1, 5, 10 and $20 \mathrm{mg} / \mathrm{ml}$, with the running buffer of the size exclusion chromatography used as reference for buffer correction. No particle interactions or radiation damage could be observed for the used data. Raw data processing was performed using the ATSAS package $^{55}$ as described in ref. 56, for example. Sets of independent $a b$ initio models were calculated using GASBOR ${ }^{57}$, and DAMAVER $^{58}$ was used for alignment and averaging. Modeling of the calcium-bound state of Twitch- 0 and Twitch- 1 was done using CORAL ${ }^{59}$ with ECFP (PDB: 2WSN), Citrine (PDB: 3DPX), the minimal domain structure described here and the respective SAXS data. To avoid clashes, we allowed up to five linker residues between the rigid bodies to account for the fact that the termini of the respective crystal structures might be flexible or change their conformation if the minimal domain folds upon $\mathrm{Ca}^{2+}$ binding and, thus, might also influence the conformation of the directly neighboring residues of the fluorescent proteins. Figures were prepared using USCF Chimera ${ }^{60}$.

Bacterial plate pre-screening of FRET calcium sensors. Libraries of biosensor mutants were subcloned into pRSETB (Invitrogen) and transformed into E. coli XL-1 Blue cells. The cells were plated on LB agar plates containing $50 \mu \mathrm{g} / \mathrm{ml}$ ampicillin with an average colony density of approximately $800-1,000$ colonies per plate and incubated overnight at $37^{\circ} \mathrm{C}$. After $1 \mathrm{~d}$ at $4{ }^{\circ} \mathrm{C}$, the biosensor mutants were fully mature and could be analyzed. For this purpose, colonies were blotted onto Whatman filter paper and imaged using a Lambda LS/30 Stand Alone Xenon Arc Lamp and Power Supply, a Lambda 10-2 Optical Filter Changer (both Sutter Instrument), and a CoolSNAP ES2 CCD Camera. The excitation filter used was D 436/40×, and the emission filters were D 480/40 (CFP, Cerulean3 and mTurquoise2) and HQ 535/30 (cpCitrine 174) (all from Chroma). Cell Profiler version 10415 Cell Image Analysis Software was used for automated region of interest (ROI) selection, and the acquired data was processed with MetaFluor version 7.7.0. The Whatman paper containing the colonies was sprayed with a solution of polylysine $(50 \mu \mathrm{g} / \mathrm{ml})$ and ionomycin $(50 \mu \mathrm{g} / \mathrm{ml})$ in a buffer of $30 \mathrm{mM}$ MOPS, $100 \mathrm{mM} \mathrm{KCl}, \mathrm{pH} \mathrm{7.5}$, to open the bacterial cell membrane. The whole membrane was dampened with the solution by spraying 4-5 times using 30-ml plastic spray bottles (Rotert, Germany). After 5 min of incubation and acquisition of baseline fluorescence, a solution of $100 \mathrm{mM}$ $\mathrm{CaCl}_{2}$ was sprayed on the cells. The change in FRET was acquired for $5 \mathrm{~min}$. Data from the plate screening were analyzed in MatLab R2011b, 10-20 of the best-performing colonies were picked, and plasmids were isolated for further analysis. Our selection criteria were lowest basal starting ratio, highest calcium-induced ratio change and the intersection of variants that ranked high in both categories. We identified the best-performing variants (top 1\% fraction per plate) using as selection criteria a starting ratio $R_{0}$ of $\sim 1$ under basal conditions and increased FRET change $(\Delta R / R)$ upon calcium addition.
Expression and purification of proteins. For protein expression and purification, genes encoding the Twitch biosensors were subcloned into pRSETb (Invitrogen) and transformed in E. coli BL21 cells. Purification of the protein of interest was achieved by using either His Mag Sepharose Ni magnetic beads (GE Healthcare) for small-scale purification, or Ni-NTA 30 polypropylene columns (Qiagen), both capable of chelating the $\mathrm{His}_{6}$ tag. The suspension was washed with column-washing buffer containing $20 \mathrm{mM}$ imidazole. Proteins were eluted by replacement of the bound protein with imidazole using column-elution buffer containing $250 \mathrm{mM}$ imidazole.

Calcium titration. To determine the $K_{\mathrm{d}}$ value, purified protein was used and a pre-warmed (room temperature) titration kit was applied as follows. Two stock solutions were prepared in accordance with previous literature ${ }^{61}$ : (i) for zero calcium, a solution of $1 \mathrm{ml}$ of zero-calcium buffer with one volume of protein solution ( $~ 0.2-1 \mu \mathrm{M}$ protein, directly in cuvette); (ii) for high calcium, a solution of $6 \mathrm{ml}$ of $39.8 \mu \mathrm{M}$ free calcium buffer with six volumes of protein solution. A spectral baseline was recorded using a fluorescence spectrophotometer (Varian Cary Eclipse). The excitation wavelength for the CFP/YFP-FRET pair was $432 \mathrm{~nm}$. The emission was determined in the range from $450 \mathrm{~nm}$ to $600 \mathrm{~nm}$ (all bandwidths $5 \mathrm{~nm}$ ). The $K_{\mathrm{d}}$ value was calculated by fitting a sigmoidal dose response curve to the plotted $\log _{10}$ values of the free calcium points (in $\mathrm{M}$ ) versus the normalized signal (normalized to $39.8 \mu \mathrm{M}$ free calcium). Ratio changes $(\Delta R / R)$ were calculated according to the formula

$$
\text { FRET Ratio Change }=\frac{\Delta R}{R}=\frac{{ }_{\mathrm{Ca}^{2+}} \text { saturated }}{-R_{\mathrm{Ca}}{ }^{2+} \text { free }}
$$

Stopped-flow measurements. To determine the kinetics $\left(K_{\text {off }}\right.$ and $K_{\text {on }}$ ) of the calcium indicators, a Varian Cary Eclipse fluorescence spectrophotometer fitted with an Applied Photophysics RX pneumatic drive unit was used. Two stock solutions were prepared as follows: calcium-saturated indicator $(5 \mathrm{ml}), 10 \mathrm{mM}$ MOPS, $4 \mathrm{mM} \mathrm{CaCl}_{2}, 2 \mathrm{mM} \mathrm{MgCl}_{2}, 50 \mathrm{mM} \mathrm{KCl}, \sim 0.2-1 \mu \mathrm{M}$ indicator (pH 7.5); BAPTA solution ( $5 \mathrm{ml}$ ), $10 \mathrm{mM}$ MOPS, $50 \mathrm{mM} \mathrm{KCl}$, $20 \mathrm{mM}$ BAPTA ( $\mathrm{pH} 7.5$ ). The stopped-flow experiment was carried out at room temperature $\left(\sim 23^{\circ} \mathrm{C}\right)$ and the two solutions were mixed with an injection pressure of 3.5 bar. Excitation was set to $432 \mathrm{~nm}$ (bandwidth $5 \mathrm{~nm}$ ) for CFP. Emission spectra of the two individual channels were taken in an alternated manner at $475 \mathrm{~nm}$ (for CFP, bandwidth $10 \mathrm{~nm}$ ) and $527 \mathrm{~nm}$ (for YFP, bandwidth $10 \mathrm{~nm}$ ) respectively. Acquisition time was set to $12.5 \mathrm{~ms}$, duration to $>10 \mathrm{~s}$ and mixing volume to $400 \mu \mathrm{l}$ with a mixing dead time of the instrument of $8 \mathrm{~ms}$. An average of the individual channels and the acceptor/donor ratio was calculated. The decay time $(\tau$, sec) was determined by fitting with a single- or double-exponential curve to the acceptor/donor ratio using OriginLab 7.5.

Screening of calcium biosensors in neonatal cultured primary rat hippocampal neurons. Neuronal screening was performed essentially as reported previously ${ }^{11,62}$, with minor adaptations for FRET ratiometric imaging. Genes encoding Twitch biosensors were subcloned into a hippocampal neuron screening vector pGP-SIV-hsyn1-IRES-nls-mCherry-WPRE-SV40 (hsyn1: human 
synapsin-1 promoter) using blunt-end cloning. Cultures of primary hippocampal neurons were obtained from P0 rat pups by dissection, papain-based dissociation and plating onto Matrigelcoated (BD Biosciences), 24-well glass-bottom plates (MatTek) and then cultured in DMEM/B27 medium (Invitrogen). The SIV-based lentiviral vectors containing Twitch biosensors were produced through transfection of HEK293T packaging cells in $10-\mathrm{cm}$ plates. On day 3 in vitro the hippocampal neuronal cultures were infected in vitro for $16 \mathrm{~h}$ using newly harvested lentiviral particles, and medium was replaced with DMEM/B27 with $4 \mathrm{mM}$ AraC (Invitrogen and Sigma). On day 16-18 in vitro infected neurons were stimulated using a custom-built, 24-well multiplexed field stimulator with platinum wires and imaged using an Olympus IX81 motorized, inverted microscope $(10 \times$ objective, 0.4 NA with optical filters $436 / 20$ for CFP excitation, 455 longpass (LP), 520 LP dichroic, 485/30 emission for CFP and FITC-TxRed emission for YFP; all optical filters and dichroic mirrors by Semrock); Prior Scientific H117 ProScanII motorized stage; a cyan (505 nm) LED illumination source (Cairn); and an EMCCD camera (AndoriXon 897, 34.8 frames per second). Field stimuli were delivered in $40-\mathrm{V}, 83-\mathrm{Hz}, 1-\mathrm{ms}$ pulses for the following trains: $1,2,3,5,10,20,40,80$ and 160 field stimuli. The whole system was automated using MetaMorph (MM; Molecular Devices) and MATLAB (MathWorks) software. Imaging buffer included the following (in $\mathrm{mM}$ ): $145 \mathrm{NaCl}$, $2.5 \mathrm{KCl}, 10$ glucose, $10 \mathrm{HEPES}$ ( $\mathrm{pH} 7.4$ ), $2 \mathrm{CaCl}_{2}, 1 \mathrm{MgCl}_{2}, 0.013-(2-$ carboxypiperazin-4-yl)-propyl-1-phosphonic acid (cPP, Tocris Bioscience), 0.01 6-cyano-7-nitroquinoxaline-2,3-dione (CNQX, Tocris Bioscience), 0.01 gabazine (Tocris Bioscience) and 1-methyl-4-carboxyphenylglycine (MCPG, TocrisBioscience). Images were processed and analyzed using custom software.

Transfection of primary cultured cells and dissociated neurons. Twitch indicators were delivered to primary cell cultures using Lipofectamine. $2-3 \mu \mathrm{g}$ of plasmid DNA were added to $250 \mu \mathrm{l}$ of Opti-MEM I. In parallel, $10 \mu \mathrm{l}$ of Lipofectamine 2000 were mixed with $250 \mu \mathrm{l}$ of Opti-MEM I and incubated for $5 \mathrm{~min}$ at room temperature. The two separate solutions containing DNA and Lipofectamine were mixed and incubated for an additional $20 \mathrm{~min}$ at room temperature to form vesicles of DNA. The DNALipofectamine solution was added to cells growing in $30-\mathrm{mm}$ glass bottom dishes, and the transfection was allowed to proceed for $3 \mathrm{~h}$ incubating at $37^{\circ} \mathrm{C}$ with $5 \% \mathrm{CO}_{2}$. After a $3 \mathrm{~h}$ of incubation, cells were washed with $1 \mathrm{ml}$ PBS and $2 \mathrm{ml}$ of DMEM (10\% FCS, $1 \%$ Pen/Strep) were added. Cells were allowed to recover from the treatment and to express the Twitch indicators overnight and were ready to use the next day.

Mouse preparation for in vivo imaging. All procedures were in accordance with protocols approved by the Janelia Farm Research Campus Institutional Animal Care and Use Committee and by the state governments of Baden-Württemberg and Upper Bavaria, Germany. C57bl mice (males and females) were used in all studies and were 2.5-3.5 months old at the start of experiments. Mice were anesthetized either with isoflurane $(2.5 \%$ for induction, $1.25-1.75 \%$ during surgery) or by intraperitoneal injection of a mixture of ketamine and xylazine $(80 \mu \mathrm{g}$ and $8 \mu \mathrm{g}$ per gram of body weight, respectively. In all cases, an animal was excluded if the cranial window quality was low.
For data shown in Figure 4a-e and Supplementary Figure 11, after skin removal and thinning of the skull, AAV encoding Twitch-3 or YC3.60 was injected through the thinned bone to the left visual cortex (2.5 and $2.9 \mathrm{~mm}$ lateral and 0.2 anterior to lambda, $250 \mu \mathrm{m}$ deep, $25 \mathrm{nl}$ per injection site). 3-4 weeks following injections, a circular craniotomy (2-3 $\mathrm{mm}$ diameter) was placed above the injection site. A custom titanium head post was fixed to the skull using black dental cement (Contemporary Ortho-Jet). The craniotomy was covered with $1 \%$ agarose and a round glass coverslip (Warner Instrument, $3 \mathrm{~mm}$ diameter, number 1 thickness) was cemented to the skull to reduce motion of the exposed brain. The animal was then transferred to the imaging setup, where it was placed on a warm blanket $\left(37^{\circ} \mathrm{C}\right)$ and kept anesthetized using $0.25-0.5 \%$ isoflurane and sedated with chlorprothixene $(20-40 \mu \mathrm{l} \text { at } 0.33 \mathrm{mg} / \mathrm{ml} \text {, intramuscular (i.m.) })^{63}$.

For data shown in Fig. 4h,i: the HIV-based lentiviruses (FUGW as the backbone, Addgene plasmid number 14883; virus titer $\geq 10^{8}$ colony-forming units per $\mathrm{ml}$ ) containing Twitch-2B or Twitch-3 biosensors were stereotactically injected $(3.0 \mathrm{~mm}$ anterior to bregma, $0.84 \mathrm{~mm}$ lateral, $2.9 \mathrm{~mm}$ deep, $\leq 1 \mu \mathrm{l}$ ) into the rostral migratory stream of C57BL/6 mice for transduction of adult-born cells moving toward the olfactory bulb. The transduced juxtaglomerular neurons in the olfactory bulb were imaged after their arrival to and settling in the glomerular layer of the olfactory bulb, at 35-37 d after infection. To study sensor function after long-term chronic expression, mice expressing Twitch-2B were imaged again at $141 \mathrm{~d}$ after infection. On the day of imaging, a mouse was anesthetized by intraperitoneal injection of a mixture of ketamine and xylazine ( $80 \mu \mathrm{g}$ and $8 \mu \mathrm{g}$, respectively, per gram of body weight), for induction; $40 \mu \mathrm{g}$ and $4 \mu \mathrm{g}$, respectively, per gram of body weight for maintenance). A custom-made recording chamber was glued to the skull. The mouse was transferred to the imaging setup, where it was placed on a warm blanket $\left(37^{\circ} \mathrm{C}\right)$. The chamber was fixed in an $x y$ table and perfused with warm $\left(38{ }^{\circ} \mathrm{C}\right.$ ) standard extracellular solution containing (in $\mathrm{mM}$ ): $125 \mathrm{NaCl}, 4.5 \mathrm{KCl}, 26 \mathrm{NaHCO}_{3}, 1.25 \mathrm{NaH}_{2} \mathrm{PO}_{4}, 2 \mathrm{CaCl}_{2}, 1 \mathrm{MgCl}_{2}$ and 20 glucose ( $\mathrm{pH} 7.4$ ) when bubbled with $95 \% \mathrm{O}_{2}$ and $5 \% \mathrm{CO}_{2}$ ). A craniotomy (typical size about $1 \mathrm{~mm} \times 0.5 \mathrm{~mm}$ ) was made using a 30-gauge syringe needle; the dura was left intact (see reference 21 for details). To reduce the movement artifacts, the craniotomy was filled with $2 \%$ agarose.

In a separate set of experiments, the area under the cranial window (prepared as described above) was stained either with Oregon Green 488 BAPTA-1 a.m. (OGB-1, Invitrogen, Carlsbad, CA) or Fura PE-3 a.m. (TEFLAB, Austin, TX) using multicell bolus loading technique ${ }^{64}$.

Odorants were applied for $4 \mathrm{~s}$ through a custom-made flowdilution olfactometer positioned in front of the snout of freely breathing mice as described previously ${ }^{21}$.

In vivo mouse imaging. For data shown in Figure $4 \mathbf{a}-\mathbf{e}$ and Supplementary Figure 11, a custom-built two-photon microscope with a resonant galvanometric scanner was used for imaging (designs available at http://research.janelia.org/Svoboda/). The light source was a Mai Tai HP 100-fs pulsed laser (SpectraPhysics) running at $860 \mathrm{~nm}$ for Twitch-3 and at $870 \mathrm{~nm}$ for YC 3.60 imaging. The objective was a $16 \times 0.8 \mathrm{NA}$ (Nikon). Image acquisition was performed using ScanImage 4 (http://www. scanimage.org $)^{65}$. Images $(512$ pixels $\times 512$ pixels, $250 \mu \mathrm{m}$ 
$\times 250 \mu \mathrm{m}$ ) were collected at $15 \mathrm{~Hz}$ from two channels (using 505-nm longpass dichroic mirror, 480/40 filter for CFP channel, and 535/50 filter for cpCitrine channel, Chroma). Mice were randomly chosen to be injected with Twitch-3 or YC3.60. The investigator was blinded to the group allocations for injection of the given AAV. Functional imaging experiments were performed 21-30 d after viral injection. Moving gratings in eight different orientations were displayed to the right eye of lightly anesthetized mice $11,63,66$. Simultaneous two-photon imaging in the contralateral layer 2/3 neurons of V1 revealed bright cellular fluorescence expression as well as neuropil staining. Notably, Twitch-3 imaging required lower excitation intensity than YC $3.60(20 \mathrm{~mW}$ versus $45 \mathrm{~mW}$ average laser power for depth of $150 \mu \mathrm{m}$ under the brain surface) to maintain a similar SNR. Ratio images exhibit robustness to small sample movements; moreover, pixel values were more uniformly distributed than the corresponding fluorescence images pixel values. Therefore, ratio images emphasize regions with lower fluorescence signal. The fraction of responsive cells was larger for Twitch-3 than for YC 3.60 (1,264/3,633 from six animals for Twitch-3, 414/2,149 from 5 animals for YC 3.60). The majority of the responsive cells had a preferred stimulus orientation (856/1,264 for Twitch-3, 258/414 for YC 3.60; Fig. 4 and Supplementary Fig. 11) in agreement with previous studies ${ }^{11,63}$. Data for OGB-1 were obtained from three animals (fraction of responsive cells was 1,297/3,606).

For data shown in Fig. $\mathbf{4 h}$,i, imaging was performed with a customized two-photon microscope based on Olympus FV1000 system (Olympus, Tokyo, Japan) and MaiTai Deep See Laser (Spectra Physics, Mountain View, CA). In this case the experimenter was not blinded to the type of GECI injected. Ten mice in total were used for this analysis with between 10 and 40 experiments performed for each indicator. The emitted light was collected with the Zeiss $20 \times$ water immersion objective (NA 1.00), and separated with a 515 LP dichroic mirror and two band-pass filters: 475/64 and 500LP for CFP and YFP channels respectively. Images were collected at $7-20 \mathrm{~Hz}$ (depending on the size of the imaging frame).

Mouse visual stimuli. Moving grating stimuli were shown to mice from the Psychophysics Toolbox ${ }^{67,68}$ generated in MATLAB. Each trial consisted of a 4-s blank stimulus period (uniform gray at mean luminance) followed by $4 \mathrm{~s}$ of drifting sinusoidal grating stimulus ( 0.05 cycles per degree, $1 \mathrm{~Hz}$ temporal frequency). The visual stimuli were synchronized to individual image frames using frame-start pulses provided by ScanImage 4 . The gratings were shown on an LCD monitor $(30 \mathrm{~cm} \times 40 \mathrm{~cm}) 25 \mathrm{~cm}$ in front of the center of the right eye of the mouse. The monitor subtended an angle of $\pm 38^{\circ}$ horizontally and $\pm 31^{\circ}$ vertically around the mouse's eye.

Image analysis. Mouse visual cortex. Analyses were performed in MATLAB. ROIs corresponding to cell bodies were chosen using a semiautomated algorithm ${ }^{11}$. Ring-shaped or circular ROIs were placed at cell cytosolic regions (excluding the nucleus) or on cell somata, respectively, according to each cell's staining pattern. Fluorescence traces for each cell were measured by averaging all pixels within the ROI. The neuropil contamination was corrected as previously described ${ }^{66}$; briefly, the neuropil signal $F_{\text {neuropil }}(t)$ surrounding each cell was measured by averaging the pixel signals within a $20-\mu \mathrm{m}$ radius from the cell center (excluding all selected cells). The true fluorescence signal of a cell body was then estimated as follows:

$$
F_{\text {cell_true }}(t)=F_{\text {cell_measured }}(t)-r \cdot F_{\text {neuropil }}(t)
$$

with $r=0.7$ used throughout the study. After neuropil correction, the $\Delta R / R_{0}$ of each trial was calculated as $\left(\mathrm{R}-\mathrm{R}_{0}\right) / \mathrm{R}_{0}$, where $\mathrm{R}_{0}$ is the baseline ratio between fluorescence signal from cpCitrine and CFP channels, averaged over a 2-s period immediately before the start of sensory stimulation, and $\mathrm{R}$ is the ratio between these two channels in each time point. Visually responsive neurons were defined as cells with $\Delta \mathrm{R} / \mathrm{R}_{0}>0.05$ during at least one stimulus period, and using ANOVA across blank and eight direction periods (averaged over $2 \mathrm{~s}, P<0.01)^{69}$.

Olfactory bulb. Data inspection during the experiment was carried out using Fluoview (Olympus, Tokyo, Japan), and NeuroPlex (RedShirtImaging LLC, Decatur, GA) software. Detailed data analysis was performed offline using a combination of Fluoview, NeuroPlex, ImageJ (http://rsb.info.nih.gov/ij/) with the WCIF plug-in (Wright Cell Imaging Facility, Toronto, Canada), MetaMorph (Molecular Devices, West Chester, PA) and Excel (Microsoft, Redmond, WA), as well as custom-made programs written for Labview (National Instruments, Austin, TX), Igor Pro (WaveMetrics, Portland, OR) or IDL (ITT, Boulder, CO).

Signal-to-noise ratios were measured as mean peak amplitude of the transients divided by the s.d. of the baseline noise.

Whole-cell recordings. Whole-cell recordings were made from layer 2/3 pyramidal cells using an EPC-10 patch-clamp amplifier (HEKA, Lambrecht, Germany) as described previously ${ }^{70}$. The HIV-based lentiviruses (FUGW as the backbone, Addgene 14883; virus titer $\geq 10^{8}$ colony forming units/ml) encoding for Twitch-2B were stereotactically injected $(2 \mathrm{~mm}$ posterior to bregma, $1.5 \mathrm{~mm}$ lateral, $0.5 \mathrm{~mm}$ deep, $\leq 1 \mu \mathrm{l}$ ) into the cortex. 1-2 weeks after viral transfection, animals were decapitated, and coronal cortical slices of $300-\mu \mathrm{m}$ thickness were cut in ice-cold standard extracellular solution. Slices were incubated in this solution for at least $1 \mathrm{~h}$ before being transferred to the setup. Patch pipettes were made from borosilicate glass (Hilgenberg $\mathrm{GmbH}$, Malsfeld, Germany) and had resistances of 7-10 MOhm when filled with the intracellular pipette solution containing (in $\mathrm{mM}$ ): 140 Potassium gluconate, $12 \mathrm{KCl}, 4 \mathrm{NaCl}, 4 \mathrm{Mg}$-ATP, $0.4 \mathrm{Na}$-GTP and 10 HEPES (pH 7.3). Experiments were performed at $35^{\circ} \mathrm{C}$. Membrane potential was held at $-70 \mathrm{mV}$. Single action potentials and bursts thereof were evoked by depolarizing current steps of different duration (5-300 ms).

Mice for functional imaging of T lymphocytes. C57BL/6 and OT-II mice ${ }^{40}$ were bred in the facilities of the Max Planck Institute of Neurobiology. All animal procedures were in accordance with the guidelines of the committee on animals of the Max Planck Institute of Neurobiology and were approved by the state government of Upper Bavaria, Germany.

Retrovirus supernatant production. The replication-deficient ecotropic retrovirus producer cells (Phoenix) were transfected using the calcium-phosphate/chloroquine method with $12 \mu \mathrm{g}$ of pMSCV retroviral vector and $3.5 \mu \mathrm{g}$ of pCL-Eco packaging 
plasmid. After $48 \mathrm{~h}$ the virus-containing culture supernatant was collected, replaced with fresh culture medium and concentrated by centrifugation at $6,000 \mathrm{RCF}$ for $18 \mathrm{~h}$ at $4{ }^{\circ} \mathrm{C}$. Pellets were resuspended in fresh virus supernatant from the transfected Phoenix cells and filtered through a $0.45-\mu \mathrm{m}$ pore-sized filter, and the filtrate was immediately used for the retroviral transduction of target cells or snap frozen on dry ice and stored at $-80^{\circ} \mathrm{C}$.

Retroviral transduction of T lymphocytes. Single-cell suspensions were prepared from spleens by mechanical disruption by forcing the spleen through $40-\mu \mathrm{m}$ cell strainers (BD Biosciences) followed by erythrocyte lysis using hypotonic $0.83 \% \mathrm{NH}_{4} \mathrm{Cl}$. For the transduction of wild-type $\mathrm{T}$ lymphocytes, $\mathrm{CD} 4^{+}$cells were purified using the MagCellect $\mathrm{CD}^{+}$cell Isolation Kit (R\&D) and were stimulated using plate-bound anti-CD3 and anti-CD28 (BD Biosciences). After $24 \mathrm{~h}$, the $\mathrm{T}$ lymphocytes were suspended in retrovirus supernatant supplemented with $8 \mu \mathrm{g} / \mathrm{ml}$ polybrene (Sigma-Aldrich) and $10 \mathrm{ng} / \mathrm{ml} \mathrm{IL-2} \mathrm{(PeproTech),} \mathrm{seeded} \mathrm{onto}$ RetroNectin-coated (Takara) wells, and spin-infected by centrifugation at $450 \mathrm{RCF}$ for $90 \mathrm{~min}$ at room temperature. For the transduction of TCR transgenic T lymphocytes, OT-II splenocyte suspensions were cultured in presence of $10 \mu \mathrm{g} / \mathrm{ml} \mathrm{OVA} 323-339$ peptide. After 48 h, Dynabeads Mouse Pan B (B220) (Invitrogen) were used to deplete the cultures of B cells, followed by transduction as described above.

T lymphocyte transfer for lymph node imaging. One day after retroviral transduction, $5 \times 10^{6}-15 \times 10^{6}$ OT-II T cells were adoptively transferred via i.v. injection into the tail vein of mildly irradiated (20 Gy) C57BL/6 mice. The mice were allowed to recover for one week before the adoptive transfer of antigen-presenting cells. Bone marrow dendritic cells (BMDCs) were obtained from the femurs of C57BL/6 mice and were cultured in the presence of GM-CSF producing hybridoma-conditioned medium with repeated medium exchange to deplete the nonadherent cells. After $8 \mathrm{~d}$ in culture, the BMDCs were trypsinized and activated overnight in fresh medium supplemented with $1 \mu \mathrm{g} / \mathrm{ml}$ LPS. The activated BMDCs were labeled using $4 \mu \mathrm{M}$ SNARF-1 (Invitrogen), and $2 \times 10^{6}$ BMDCs were injected subcutaneously into the lower leg. Imaging of the draining popliteal lymph node was performed $1 \mathrm{~d}$ later.

In vivo two-photon microscopy calcium imaging of $\mathrm{T}$ lymphocytes. Time-lapse two-photon laser-scanning microscopy was performed essentially as reported previously ${ }^{36}$ using an SP2 confocal microscope (Leica) equipped with a $10 \mathrm{~W}$ Millenia/ Tsunami laser (Newport Spectra Physics). The laser wavelength was set to $835 \mathrm{~nm}$ for CFP excitation and directed through a Leica $25 \times$ water immersion objective (NA 0.95). Areas measuring $240 \mu \mathrm{m} \times 240 \mu \mathrm{m}$ were scanned, and $z$-stacks $(25-30 \mu \mathrm{m})$ were acquired using a 3 - to 4 - $\mu \mathrm{m} z$-step. The acquisition rate was at intervals of 16-20 s, with images averaged twice. The fluorescence signals were recorded using non-descanned photomultiplier tube (PMT) detectors (Hamamatsu) equipped with 475/50 nm (CFP), 537/26 nm (FRET), and 630/69 nm (SNARF-1, Texas Red) band-pass filters (Semrock). The PMT settings for CFP and FRET fluorescence were kept identical during all experiments. To estimate FRET ratios, CFP bleed-through was corrected. The CFP bleed-through into the FRET channel was determined to $44 \%$. The fraction of cpCitrine emission in the CFP channel and direct excitation of cpCitrine at $835 \mathrm{~nm}$ was negligible. The mice were anaesthetized by i.p. injection with fentanyl, midazolam, medetomidine $(50 \mu \mathrm{g}, 50 \mathrm{mg}$, and $500 \mu \mathrm{g}$ per kilogram of body weight, respectively), intubated and ventilated with $1.5 \%$ isoflurane. To expose the popliteal lymph nodes the skin was cut at the hollow of the knee followed by a careful dissection of the adductor musculature. Animals were stabilized in a custom-made microscope stage, and the body temperature was regulated using a heat pad $\left(37.5^{\circ} \mathrm{C}\right)$. Physiological parameters and electrocardiograms were constantly monitored and recorded during the imaging. Blood vessels were visualized by the i.v. infusion of Texas Redconjugated dextran (50 $\mu \mathrm{g} ; 70 \mathrm{kDa}$; Invitrogen).

Imaging data analysis (T lymphocytes). Images were acquired using Leica LCS software and subsequently processed and analyzed by ImageJ (http://rsb.info.nih.gov/ij/). A Gaussian blur filter was applied and maximum intensity $z$-projections were made to obtain two-dimensional videos. In the images shown in Figure 5, the CFP channel is depicted as green and the YFP channel as red. For analysis, cell shapes at each time point were manually outlined in the maximum projection picture, and the average signal intensities of all pixels for the area were calculated. The calculation of motility and calcium signals was done using Excel (Microsoft). The FRET signal was corrected for CFP bleed-through, cFRET = FRET $-0.44 \times$ CFP, and the ratios of cFRET/CFP were normalized to display the fractional ratio change $\Delta \mathrm{R} / \mathrm{R} 0$ ( $\mathrm{R} 0$ for Twitch-1 $=0.8$; Twitch-2B 0.5). Values were arbitrarily chosen for Twitch-1 so that only a tiny fraction of cells would be lower than this threshold. For Twitch-2B, R0 was adjusted to match the baseline population to the same level than Twitch-1). The overlaid box plots on the scatter plots in Figure 5 indicate the 25th and 75th percentiles, and the whiskers represent the 5th and 95th percentiles. All statistical analysis (Mann-Whitney $U$ test) was performed with GraphPad Prism.

41. Ikura, M., Kay, L.E. \& Bax, A. A novel approach for sequential assignment of $1 \mathrm{H}, 13 \mathrm{C}$, and $15 \mathrm{~N}$ spectra of proteins: heteronuclear triple-resonance three-dimensional NMR spectroscopy. Application to calmodulin. Biochemistry 29, 4659-4667 (1990).

42. Grzesiek, S. \& Bax, A. An efficient experiment for sequential backbone assignment of medium sized isotopically enriched proteins. J. Magn. Reson. 99, 201-207 (1992).

43. Grzesiek, S. \& Bax, A. Correlating backbone amide and sidechain resonances in larger proteins by multiple relayed triple resonance NMR. J. Am. Chem. Soc. 114, 6291-6293 (1992).

44. Vuister, G.W. \& Bax, A. Quantitative J correlation: a new approach for measuring homonuclear three bond $\mathrm{J}(\mathrm{HNHa})$ coupling constants in $15 \mathrm{~N}$-enriched proteins. J. Am. Chem. Soc. 115, 7772-7777 (1993).

45. Hansen, M.R., Mueller, L. \& Pardi, A. Tunable alignment of macromolecules by filamentous phage yields dipolar coupling interactions. Nat. Struct. Biol. 5, 1065-1074 (1998).

46. Ottiger, N., Delaglio, F. \& Bax, A. Measurement of $\mathrm{J}$ and dipolar couplings from simplified two-dimensional NMR spectra. J. Magn. Reson. 131, 373-378 (1998).

47. Delaglio, F. et al. NMRPipe: a multidimensional spectral processing system based on UNIX pipes. J. Biomol. NMR 6, 277-293 (1995).

48. Keller, R.L.J. The Computer Aided Resonance Assignment Tutorial (Cantina, 2004).

49. Shen, Y. et al. Consistent blind protein structure generation from NMR chemical shift data. Proc. Natl. Acad. Sci. USA 105, 4685-4690 (2008).

50. Raman, S. et al. NMR structure determination for larger proteins using backbone-only data. Science 327, 1014-1018 (2010).

51. Koradi, R., Billeter, M. \& Wuthrich, K. MOLMOL: a program for display and analysis of macromolecular structures. J. Mol. Graph. 14, 51-55 (1996). 
52. Pettersen, E.F. et al. UCSF Chimera-a visualization system for exploratory research and analysis. J. Comput. Chem. 25, 1605-1612 (2004).

53. Laskowski, R.A., Rullmannn, J.A., MacArthur, M.W., Kaptein, R. \& Thornton, J.M. AQUA and PROCHECK-NMR: programs for checking the quality of protein structures solved by NMR. J. Biomol. NMR 8, 477-486 (1996).

54. Davis, I.W. et al. MolProbity: all-atom contacts and structure validation for proteins and nucleic acids. Nucleic Acids Res. 35, W375-W383 (2007).

55. Konarev, P.V., Petoukhov, M.V., Volkov, V.V. \& Svergun, D.I. ATSAS 2.1, a program package for small-angle scattering data analysis. J. Appl. Cryst. 39, 277-286 (2006).

56. Putnam, C.D., Hammel, M., Hura, G.L. \& Tainer, J.A. X-ray solution scattering (SAXS) combined with crystallography and computation: defining accurate macromolecular structures, conformations and assemblies in solution. Q. Rev. Biophys. 40, 191-285 (2007).

57. Svergun, D.I., Petoukhov, M.V. \& Koch, M.H.J. Determination of domain structure of proteins from X-ray solution scattering. Biophys. J. 80, 2946-2953 (2001).

58. Volkov, V.V. \& Svergun, D.I. Uniqueness of $a b$ initio shape determination in small-angle scattering. J. Appl. Cryst. 36, 860-864 (2003).

59. Petoukhov, M.V. et al. New developments in the ATSAS program package for small-angle scattering data analysis. J. Appl. Cryst. 45, 342-350 (2012).

60. Wriggers, W. \& Chacon, P. Using Situs for the registration of protein structures with low-resolution bead models from X-ray solution scattering. J. Appl. Cryst. 34, 773-776 (2001).
61. Tsien, R. \& Pozzan, T. Measurement of cytosolic free $\mathrm{Ca}^{2+}$ with quin2. Methods Enzymol. 172, 230-262 (1989).

62. Wardill, T.J. et al. A neuron-based screening platform for optimizing genetically encoded calcium indicators. PLOS ONE 8, e77728 (2013).

63. Niell, C.M. \& Stryker, M.P. Highly selective receptive fields in mouse visual cortex. J. Neurosci. 28, 7520-7536 (2008).

64. Stosiek, C., Garaschuk, 0., Holthoff, K. \& Konnerth, A. In vivo two-photon calcium imaging of neuronal networks. Proc. Natl. Acad. Sci. USA 100, 7319-7324 (2003).

65. Pologruto, T.A., Yasuda, R. \& Svoboda, K. Monitoring neural activity and $\left[\mathrm{Ca}^{2+}\right]$ with genetically encoded $\mathrm{Ca}^{2+}$ indicators. J. Neurosci. 24 9572-9579 (2004).

66. Kerlin, A.M., Andermann, M.L., Berezovskii, V.K. \& Reid, R.C. Broadly tuned response properties of diverse inhibitory neuron subtypes in mouse visual cortex. Neuron 67, 858-871 (2010).

67. Brainard, D.H. The psychophysics toolbox. Spat. Vis. 10, 433-436 (1997).

68. Pelli, D.G. The VideoToolbox software for visual psychophysics: transforming numbers into movies. Spat. Vis. 10, 437-442 (1997).

69. Ohki, K., Chung, S., Ch'Ng, Y.H., Kara, P. \& Reid, R.C. Functional imaging with cellular resolution reveals precise micro-architecture in visual cortex. Nature 433, 597-603 (2005).

70. Garaschuk, 0., Hanse, E. \& Konnerth, A. Developmental profile and synaptic origin of early network oscillations in the CA1 region of rat neonatal hippocampus. J. Physiol. (Lond.) 507, 219-236 (1998). 